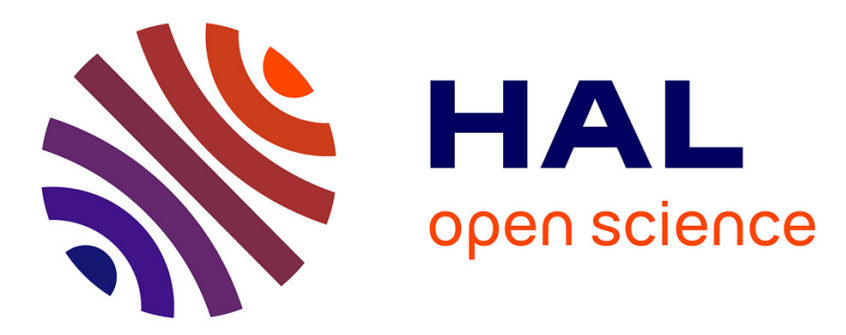

\title{
Etude et modélisation de la dégradation des transistors MOS submicroniques soumis à une contrainte électrique
}

\author{
B. Cabon-Till, G. Ghibaudo
}

\section{To cite this version:}

B. Cabon-Till, G. Ghibaudo. Etude et modélisation de la dégradation des transistors MOS submicroniques soumis à une contrainte électrique. Revue de Physique Appliquée, 1986, 21 (5), pp.305-318. 10.1051/rphysap:01986002105030500 . jpa-00245448

\section{HAL Id: jpa-00245448 https://hal.science/jpa-00245448}

Submitted on 1 Jan 1986

HAL is a multi-disciplinary open access archive for the deposit and dissemination of scientific research documents, whether they are published or not. The documents may come from teaching and research institutions in France or abroad, or from public or private research centers.
L'archive ouverte pluridisciplinaire HAL, est destinée au dépôt et à la diffusion de documents scientifiques de niveau recherche, publiés ou non, émanant des établissements d'enseignement et de recherche français ou étrangers, des laboratoires publics ou privés. 
Classification

Physics Abstracts

$73.40 \mathrm{Q}-73.90$

\title{
Etude et modélisation de la dégradation des transistors MOS submicroniques soumis à une contrainte électrique
}

\author{
B. Cabon-Till et G. Ghibaudo \\ Laboratoire de Physique des Composants à Semiconducteurs, UA-CNRS 840, ENSERG, 23 Av. des Martyrs, \\ 38031 Grenoble, France
}

(Reçu le 14 novembre 1985, révisé le 14 janvier 1986, accepté le 14 janvier 1986)

\begin{abstract}
Résumé. - Le vieillissement des TMOS submicroniques est étudié par l'évolution expérimentale de la mobilité d'effet de champ $\mu_{\mathrm{FE}}$ (transconductance normalisée en régime ohmique) au cours de contraintes de différentes durées. L'évolution de la tension de seuil extrapolée et celle de la tension de seuil de mobilité sont également étudiées. Les dégradations les plus importantes sont observées en fin de contrainte pour les plus fortes tensions de grille appliquées. Les variations du maximum de mobilité et de tensions de seuil au cours du temps montrent deux régimes : prédominance de la dégradation de mobilité en début de contrainte, et création dominante d'états d'interface et/ou de charges fixes avec diminution du taux de variation de mobilité après plusieurs heures de contrainte. Les dégradations de mobilité sont corrélées à la variation de la tension de seuil de mobilité et à celle de la tension de seuil extrapolée. Enfin, les résultats expérimentaux sont analysés grâce à un modèle de transconductance basé sur la dépendance de la mobilité avec la charge d'inversion.
\end{abstract}

\begin{abstract}
The ageing of submicron MOSTs is studied here by way of the experimental variation of the field effect mobility $\mu_{\mathrm{FE}}$ (normalized transconductance in the ohmic region) with stress time. The changes in the extrapolated threshold voltage and the mobility threshold voltage are also presented. The largest degradations were seen after a long time of ageing at the highest applied gate voltages. By examining the variation of the mobility maximum and the threshold voltages with stress time, two distinct regions were distinguished : firstly, for short stress times, one of dominant mobility degradation, and secondly for stress times of many hours, one of dominant interface states and/or oxide fixed charges generation and a lower rate of mobility variation. The mobility degradation is correlated to the variations of mobility threshold voltage and extrapolated threshold voltage. Experimental results are then analysed with a model of transconductance based on an inversion charge dependent mobility.
\end{abstract}

\section{Introduction.}

Nous présentons les effets d'une contrainte électrique de durée variable appliquée sur des transistors MOS submicroniques à canal $\mathbf{n}$ enrichi.

Les dégradations de transconductance (ou de mobilité) et de tension de seuil des transistors MOS soumis à d'importants champs électriques ont fait l'objet de nombreuses publications [1-5]. Cependant beaucoup d'avis controversés demeurent quant au rôle des trous chauds [6-8] ou des électrons chauds $[2,5,9]$ injectés dans l'oxyde pendant la contrainte.

La tendance actuelle à la réduction des dimensions des MOS entraîne une augmentation de ces dégradations dues à l'accroissement du champ électrique. Nous donnons ici une illustration de telles dégradations pour des transistors de longueurs de canal ultracourtes $0,4 \mu \mathrm{m}<L_{\mathrm{ch}}<1,4 \mu \mathrm{m}$ et ayant de fines épaisseurs d'oxyde $\left(t_{\mathrm{ox}}=26 \mathrm{~nm}\right)$ ce qui fait une des originalités de ce travail. Les difficultés dans ce cas résident dans les effets électriques de canaux ultracourts dus au caractère bidimensionnel du fonctionnement du transistor.

Nous exploitons les caractéristiques expérimentales $I_{\mathrm{D}}\left(V_{\mathrm{G}}\right)$ du TMOS après vieillissement et en régime ohmique, ce qui limite les effets liés au perçage [10] et à la dissymétrie des rôles de source et de drain. L'évolution des caractéristiques $I_{\mathrm{D}}\left(V_{\mathrm{D}}\right)$ après contrainte a déjà été présentée antérieurement [11]. Les dégradations sont localisées à une petite région près du drain, entraînant une inhomogénéité du canal : le régime ohmique ne permettra d'étudier que les valeurs moyennes, sur toute la longueur du canal, des paramètres considérés. A partir des vieillissements présentés, nous proposons un modèle analytique permettant d'interpréter les dégradations observées expérimentalement et d'expliciter les rôles que les états d'interfaces y tiennent. 
Les valeurs expérimentales $I_{\mathrm{D}}\left(V_{\mathrm{G}}\right)$ en régime ohmique permettent directement l'obtention de la conductance $G_{\mathrm{D}}=I_{\mathrm{D}} / V_{\mathrm{D}}$, et de la transconductance $G_{\mathrm{G}}=\delta I_{\mathrm{D}} / \delta V_{\mathrm{G}}$ ou de la mobilité d'effet de champ $\mu_{\mathrm{FE}}$ proportionnelle à la transconductance et normalisée aux faibles tensions de drain et aux dimensions du TMOS [12-13] :

$$
\mu_{\mathrm{FE}}=\left.\frac{G_{\mathrm{G}}}{V_{\mathrm{D}}} \frac{L_{\mathrm{ch}}}{W C_{\mathrm{ox}}}\right|_{V_{\mathrm{D}} \rightarrow 0}
$$

où $V_{\mathrm{D}}=$ tension de drain, $W=$ largeur du canal, $C_{\mathrm{ox}}=$ capacité d'oxyde de grille, $L_{\mathrm{ch}}=$ longueur du canal.

La région du point d'inflexion de la caractéristique $I_{\mathrm{D}}\left(V_{\mathrm{G}}\right)$ fournit 3 paramètres intéressants :

- $\mu_{\mathrm{m}}$ : maximum de la caractéristique $\mu_{\mathrm{FE}}\left(V_{\mathrm{G}}\right)$;

- $V_{\mathrm{Gm}}$ : tension de grille correspondante au maximum $\mu_{\mathrm{m}}$ (Fig. 1a), et choisie, par ailleurs, comme tension de seuil [14];

- $V_{\text {Text }}$ : tension de seuil obtenue par extrapolation de la tangente à la courbe $I_{\mathrm{D}}\left(V_{\mathrm{G}}\right)$ au point d'inflexion vers $I_{\mathrm{D}}=0$.

Les paramètres $\mu_{\mathrm{m}}$ et $V_{\mathrm{Gm}}$ seront choisis, par la suite, pour examiner les dégradations après vieillissement à cause de leur forte sensibilité en régime ohmique dans une petite région autour du point d'inflexion des caractéristiques de transfert.

Notons que la mobilité d'effet de champ diffère de la mobilité effective des électrons de la couche d'inversion qui, elle, se déduit de la conductance par :

$$
\mu_{\text {eff }}=\left.\frac{L_{\mathrm{ch}} G_{\mathrm{D}}}{W Q_{\mathrm{i}}}\right|_{V_{\mathrm{D}} \rightarrow 0}
$$

où $Q_{\mathrm{i}}=$ charge absolue dans la couche d'inversion.

Soulignons que la mobilité de champ est différente de la mobilité effective en régime de faible inversion et s'en rapproche, tout en lui étant inférieure, en régimes de moyenne et de forte inversion.

Les valeurs expérimentales de $\mu_{\mathrm{FE}}$ pourront être comparées aux valeurs théoriques de cette mobilité calculées à partir des relations (1) et (2) par :

$$
\mu_{\mathrm{FE}}=\frac{1}{C_{\mathrm{ox}}}\left[\frac{\delta \mu_{\mathrm{eff}}}{\delta V_{\mathrm{G}}} Q_{\mathrm{i}}+\mu_{\mathrm{eff}} \frac{\delta Q_{\mathrm{i}}}{\delta V_{\mathrm{G}}}\right]_{V_{\mathrm{D}} \rightarrow 0} .
$$

Nous obtiendrons les variations théoriques $\mu_{\mathrm{FE}}\left(V_{\mathrm{G}}\right)$ par une relation liant $Q_{\mathrm{i}}$ au potentiel de surface et à $\mu_{\text {eff }}$ [15], dans tous les régimes d'inversion du TMOS. Ceci nous permettra d'analyser les dégradations en les reliant aux créations d'états d'interface d'une part, et aux fluctuations de potentiel de surface d'autre part. Nous montrerons ainsi que la dégradation de mobilité ne peut pas s'expliquer seulement par une augmentation de la densité d'états d'interface comme cela a déjà été publié $[2,3,5]$. De même, nous analyserons la corrélation entre la dégradation du maximum de mobilité $\mu_{m}$ et celles de la tension de

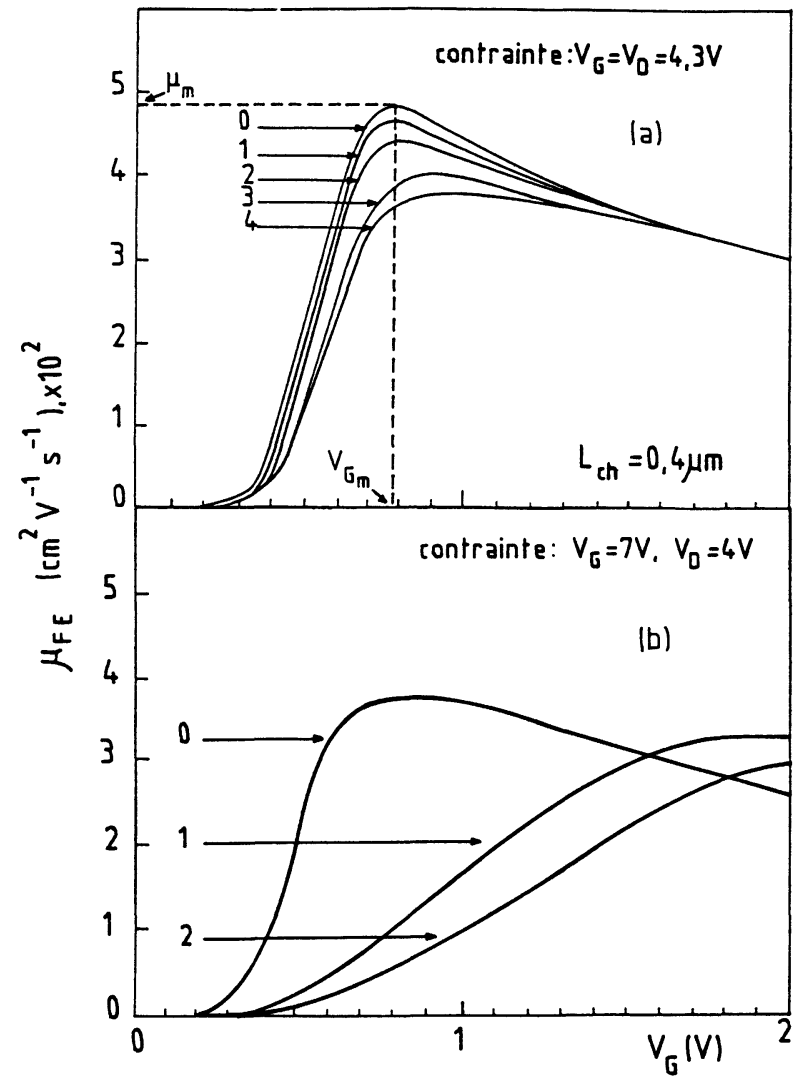

Fig. 1. - Variation expérimentale de $\mu_{\mathrm{FE}}$ (mobilité d'effet de champ) avec la tension de grille $V_{\mathrm{G}}$. Caractéristiques typiques de la dégradation : caractéristique initiale (0), après $2 \mathrm{~h}(1), 10 \mathrm{~h}(2), 50 \mathrm{~h}(3), 70 \mathrm{~h}(4)$ de contrainte $V_{G}=V_{D}$ $\left(\mathrm{T}_{2}\right.$, Fig. 1a) et $V_{\mathrm{G}}>V_{\mathrm{D}}\left(\mathrm{T}_{3}\right.$, Fig. 1b) appliquées à un TMOS à très court canal.

[Experimental variation of $\mu_{\mathrm{FE}}$ (field effect mobility) with gate voltage $V_{\mathrm{G}}$. Typical characteristics of device degradation; initial curve (0), after 2 hours (1), 10 hours (2), 50 hours (3), 70 hours (4) of stress with $V_{G}=V_{D}\left(\mathrm{~T}_{2}\right.$, Fig. 1a) and $V_{G}>V_{D}\left(T_{3}\right.$, Fig. 1b) applied on a very short channel MOST.]

seuil de mobilité $V_{\mathrm{Gm}}$ associée et de la tension de seuil $V_{\text {Text }}$.

Les modèles considérés bien qu'unidimensionnels rendront bien compte de la plupart des effets perçus $\mathrm{au}$ cours $\mathrm{du}$ vieillissement.

\section{Conditions expérimentales.}

Les TMOS submicroniques étudiés ont été fabriqués au LETI (Grenoble) selon un processus technologique décrit par ailleurs [11].

Les dispositifs ont été vieillis à température ambiante, avec source et substrat reliés à la masse. Nous avons étudié l'influence de différentes conditions de polarisation : $V_{\mathbf{G}}<V_{\mathrm{D}}$ (condition de maximum de courant de substrat), $V_{\mathrm{G}}=V_{\mathrm{D}}$ (condition de maximum de courant de grille), et $V_{\mathrm{G}}>V_{\mathrm{D}}$. Celles-ci ont été appliquées sur des transistors de différentes 
Tableau I. - Conditions expérimentales relatives aux contraintes appliquées.

[Experimental conditions of applied electrical stress.]

Transistor Longueur de canal $\begin{gathered}\text { Conditions } \\ \text { de contrainte }\end{gathered}$

\begin{tabular}{lll}
\hline $\mathrm{T}_{1}$ & $\sim 0,4 \mu \mathrm{m}$ & $V_{\mathrm{G}}=V_{\mathrm{D}} / 2$ \\
$\mathrm{~T}_{2}$ & $\sim 0,4 \mu \mathrm{m}$ & $V_{\mathrm{G}}=V_{\mathrm{D}}$ \\
$\mathrm{T}_{3}$ & $\sim 0,4 \mu \mathrm{m}$ & $V_{\mathrm{G}}>V_{\mathrm{D}}$ \\
$\mathrm{T}_{4}$ & $\sim 1,4 \mu \mathrm{m}$ & $V_{\mathrm{G}}=V_{\mathrm{D}}$ \\
$\mathrm{T}_{5}$ & $\sim 1,4 \mu \mathrm{m}$ & $V_{\mathrm{G}}>V_{\mathrm{D}}$
\end{tabular}

longueurs de canal et peuvent être résumées selon le tableau I. La tension de drain est maintenue constante $\left(V_{\mathrm{D}} \# 4,5 \mathrm{~V}\right)$ et correspondant au régime de fonctionnement du TMOS en préavalanche : $V_{\mathrm{D}} \sim V_{\mathrm{a}}$ où $V_{\mathrm{a}}$ est la valeur critique définie comme la tension de drain au-dessus de laquelle on a une augmentation de $10 \%$ de la conductance en régime de saturation. La tension de grille, toujours positive, n'excède pas $7 \mathrm{~V}$ pour éviter le claquage de l'oxyde.

Les mesures des caractéristiques courant-tension sont réalisées sur un banc automatique comportant un picoampèremètre $\mathrm{HP} 4140 \mathrm{~B}$ et un microcalculateur HP9826.

\section{Résultats expérimentaux.}

Pour l'extraction des principaux paramètres des TMOS, nous utilisons une méthode proposée antérieurement [14] qui assure la détermination du maximum de mobilité $\mu_{\mathrm{m}}$, de la tension de grille correspondante $V_{\mathrm{Gm}}$ et du coefficient de réduction de mobilité $\theta$ en s'affranchissant des effets dus à la résistance série de source et de drain $R_{\mathrm{SD}}$ [16], $\theta$ étant défini en inversion forte par :

$$
\mu_{\text {eff }}=\frac{\mu_{0}}{\left[1+\theta\left(V_{\mathrm{G}}-V_{\mathrm{T}}\right)\right]}
$$

où $\mu_{\text {eff }}=$ mobilité effective, $V_{\mathrm{T}}=$ tension de seuil de la charge d'inversion [14], différente de $V_{\text {Text }}$ [14].

$\mathrm{Au}$ cours des vieillissements étudiés, le facteur $\theta$ subit au plus une diminution de $10 \%$. L'évolution des autres paramètres $\Delta \mu_{\mathrm{m}} / \mu_{\mathrm{m}}, \Delta V_{\mathrm{Gm}}, \Delta V_{\text {Text }}$ est plus sensible au vieillissement.

La figure 1a montre, à partir des courbes $\mu_{\mathrm{FE}}\left(V_{\mathrm{G}}\right)$, l'évolution des paramètres $V_{\mathrm{Gm}}$ et $\mu_{\mathrm{m}}$ au cours de 4 étapes de vieillissement du transistor $T_{2}$. Ces caractéristiques montrent que la dégradation a lieu principalement autour du maximum $\mu_{\mathrm{m}}$ de mobilité correspondant à un régime de fonctionnement du transistor dans la transition entre l'inversion faible et forte. On constate une faible variation des tensions de seuil $V_{\mathrm{Gm}}$ et $V_{\text {Text }}$. Ce type de dégradation est attribué principalement à une réduction de la mobilité dans le canal, et a été interprété par une création d'états d'interface induite par une injection d'électrons chauds du canal (C.H.E.) plutôt que par une augmentation de charges piégées dans l'oxyde [2]. La figure $1 \mathrm{~b}$ montre la caractéristique $\mu_{\mathrm{FE}}\left(V_{\mathrm{G}}\right)$ d'un transistor $\mathrm{T}_{3}$ après vieillissement. On remarque ici par contre une très forte variation de $V_{\mathrm{Gm}}$ dès le début de la contrainte.

L'évolution au cours du temps des paramètres $\mu_{\mathrm{m}}$, $V_{\mathrm{Gm}}$ et $V_{\text {Text }}$ est représentée avec une échelle semilogarithmique sur les figures $2 \mathrm{a}$ et $2 \mathrm{~b}$ respectivement pour des transistors $\mathrm{T}_{2}$ et $\mathrm{T}_{5}$. La variation de la tension de seuil extrapolée $\Delta V_{\text {Texı }}$, très faible (cas de $\mathrm{T}_{2}$ ) et parfois négative (cas de $T_{5}$ ) au début du vieillissement, s'accélère ensuite [1], ce que nous expliquerons par la suite. La variation de $V_{\mathrm{Gm}}$ garde, comme $\mu_{\mathrm{m}}$, toujours le même signe et est bien plus grande que celle de $V_{\text {Text }}$. A ce titre, $V_{\mathrm{Gm}}$ apparaît comme un paramètre très sensible au vieillissement. On peut noter que $V_{\mathrm{Gm}}$ et $V_{\text {Text }}$ varient proportionnellement dans le cas de plus fortes contraintes (cas de $\mathrm{T}_{2}$ apparaissant sur la Fig. 2a et de $T_{3}$ ).
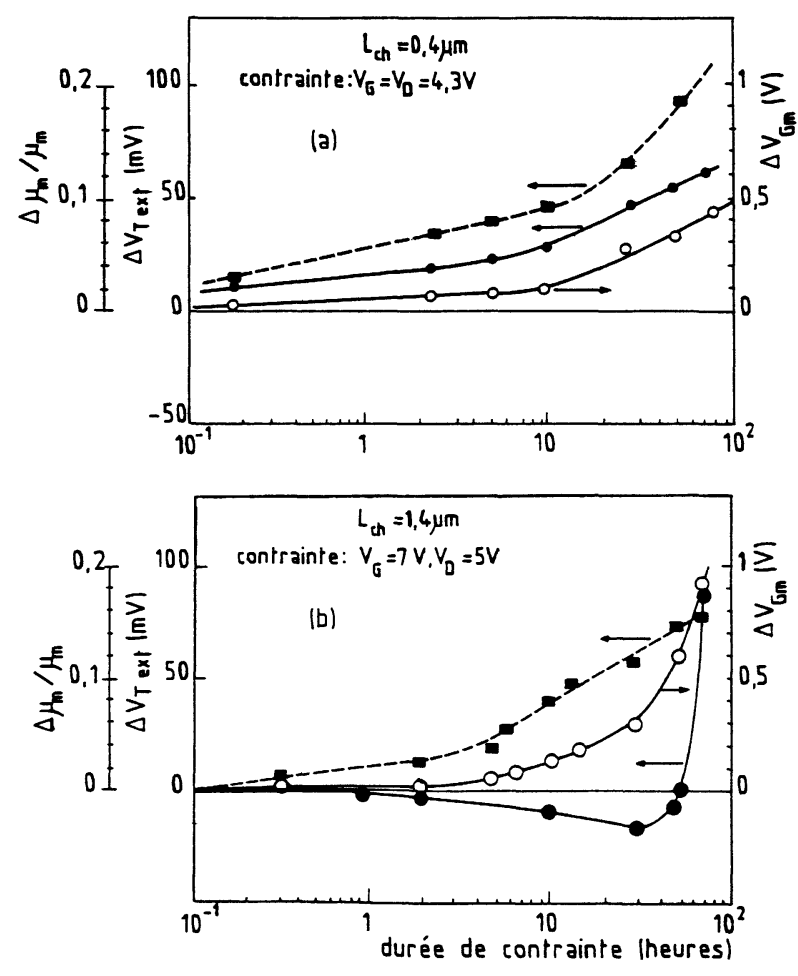

Fig. 2. - Evolution au cours du temps de la variation relative du maximum de mobilité $\mu_{\mathrm{m}}(--)$ et de la variation de la tension de seuil $V_{\text {Text }}(\longrightarrow-$ ) (échelle de gauche), ainsi que celle de la tension de seuil $V_{\mathrm{Gm}}(-\mathrm{O}-$ ) (échelle de droite), pour un TMOS à très court canal soumis à la contrainte $V_{\mathrm{G}}=V_{\mathrm{D}}$ (Fig. 2a), et un TMOS à canal « long» soumis à la contrainte $V_{G}>V_{D}$ (Fig. 2b).

[Curves of the relative variation of the mobility maximum $\mu_{\mathrm{m}}(--)$ and absolute variations of the threshold voltages $V_{\text {Text }}(\longrightarrow-)$ (left scale) and $V_{\mathrm{Gm}}(-\mathrm{O} \longrightarrow$ (right scale) with stress time for a very short channel MOST stressed under the bias condition $V_{G}=V_{D}$ (Fig. 2a) and for a «long" channel MOST stressed under the bias condition $V_{\mathrm{G}}>V_{\mathrm{D}}$ (Fig. 2b).] 
La figure 3a montre, sur une échelle logarithmique, que le modèle de variation au cours du temps de transconductance et de tension de seuil proposé par Takeda [3,17] et Chen [4] s'applique aux variations de la tension de seuil de mobilité $V_{\mathrm{Gm}}$ pour des durées de vieillissement relativement courtes : $V_{\mathrm{Gm}} \sim t^{n}$; la pente $n$ dépend fortement de la tension de grille appliquée lors de la contrainte [3,17], et prend la valeur 1 lorsque $V_{\mathrm{G}}=7 \mathrm{~V}$, et 0,25 lorsque $V_{\mathrm{G}}=4,8 \mathrm{~V}$, pour un transistor dont le canal est relativement long $\left(T_{4}\right.$ et $\left.T_{5}\right)$. Il apparaît sur les figures $3 a$ et $3 b$ que cette loi s'applique aussi aux variations du maximum de mobilité $\mu_{\mathrm{m}}$ dans la limite de faibles dégradations (faibles tensions de contrainte et/ou faibles durées) : pour de longues durées de vieillissement apparait une diminution du taux de variation de la mobilité [17-18]. Ceci permet de distinguer deux régimes de vieillissement :

- le premier, pour de faibles durées de contrainte s'accompagne d'une dégradation prédominante de la mobilité;

- le deuxième, après de longues durées de contrainte, est marqué par une dégradation prédominante de tensions de seuils liée à une plus forte création d'états d'interface et/ou de charges négatives dans l'oxyde.

Les figures $3 \mathrm{a}$ et $3 \mathrm{~b}$ montrent également, pour les deux types de longueur étudiés, que les dégradations consécutives à $100 \mathrm{~h}$ de contrainte sont plus importantes pour les tensions de grille appliquées les plus fortes lors de la contrainte. Toutefois, pour des durées de contrainte inférieures à $10 \mathrm{~h}$, la dégradation du maximum de mobilité peut être plus importante pour des tensions de grille appliquées faibles comme cela a déjà été observé [17]. Pour les durées de vieillissement envisagées ici (100 $\mathrm{h}$ au maximum), on peut noter que les transistors de plus grandes longueurs de canal ne vieillissent pas pour les tensions de grille appliquées faibles : $V_{\mathrm{G}}<V_{\mathrm{D}}$. Cette condition, qui correspond pourtant au maximum de courant de substrat pour ces transistors, est souvent considérée comme la plus efficace $[1,17,19]$.

Les figures $4 \mathrm{a}$ et $4 \mathrm{~b}$ représentent les dégradations de la caractéristique de transfert $\log I_{\mathrm{D}}\left(V_{\mathrm{G}}\right)$ en inversion faible au cours de différentes étapes du vieillissement, pour différentes longueurs de canal et différentes contraintes. La figure $4 \mathrm{a}$ compare l'effet d'une même contrainte appliquée $\left(V_{\mathrm{G}}=V_{\mathrm{D}}\right)$ à deux transistors de longueurs de canal différentes, et montre une dégradation plus prononcée pour les canaux les plus courts. Pour ces derniers, l'augmentation de la pente $S=$ $\delta \log \left(I_{\mathrm{D}}\right) / \delta V_{\mathrm{G}}$ de cette caractéristique s'oppose aux effets habituels dus à la création d'états d'interface : cette pente ne peut permettre d'évaluer correctement la densité d'états d'interface [20] car elle dépend aussi du taux de fluctuations de charge à l'interface ce qui entraîne une mobilité effective réduite en régime de faible inversion, inférieure à la mobilité microscopique [21], et non constante dans ce régime; cet effet

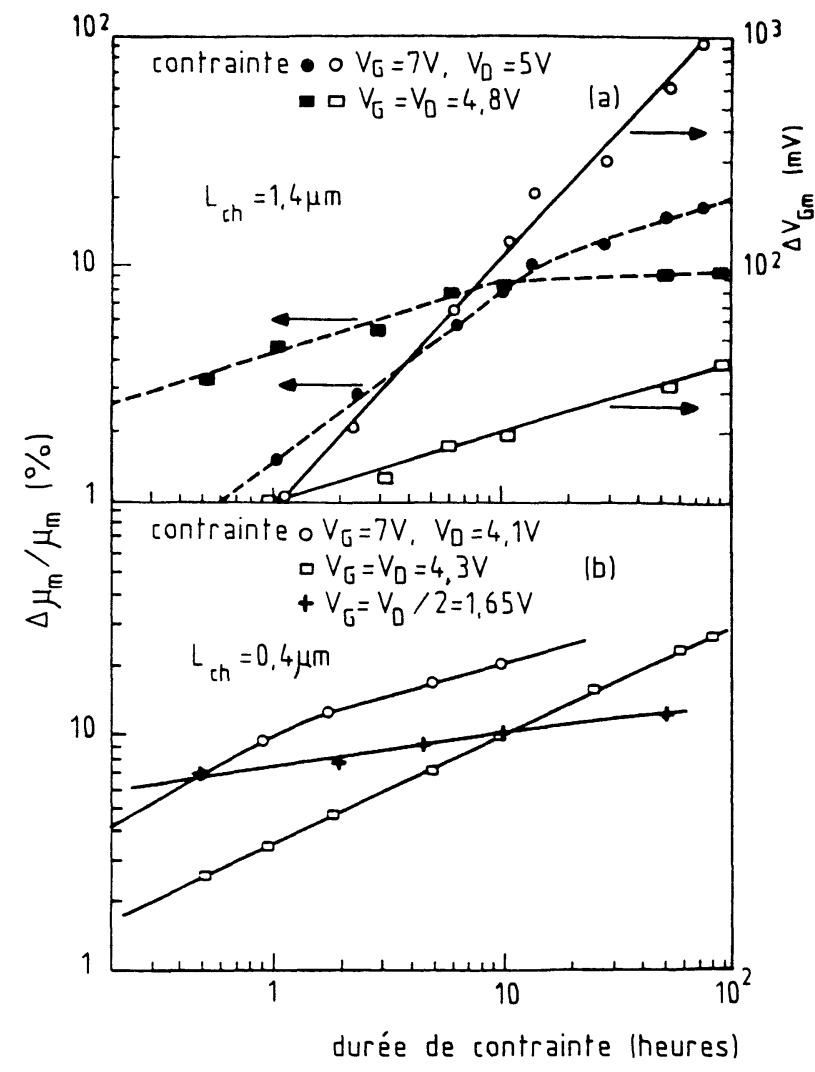

Fig. 3. - a) Variation des paramètres $V_{\mathrm{Gm}}(\longrightarrow$ (échelle de droite) et $\mu_{\mathrm{m}}(---)$ (échelle de gauche) au cours du temps, en échelle logarithmique, pour deux contraintes différentes appliquées à un TMOS à canal «long». b) Variation relative du maximum de mobilité $\mu_{\mathrm{m}}$ au cours du temps en échelle logarithmique, pour trois contraintes appliquées à un TMOS à canal très court.

[a) Log-log plot of $\Delta V_{\mathrm{Gm}}\left(\longrightarrow\right.$ (right scale) and $\Delta \mu_{\mathrm{m}} / \mu_{\mathrm{m}}$ (- - -) left scale) for a « long » channel device stressed under two bias conditions. b) Log-log plot of the relative shift in $\mu_{\mathrm{m}}$ with time for a very short channel device stressed under three bias conditions.]

peut conduire à une interprétation erronée de la pente $S$ en inversion faible. La figure $4 \mathrm{~b}$ montre également que, pour une contrainte $V_{\mathrm{G}}=V_{\mathrm{D}} / 2$ appliquée à un transistor de canal très court, la pente $S$ diminue en début de contrainte (forte dégradation dans les premières minutes déjà observée sur la Fig. 3b) avec une augmentation du courant, pour augmenter ensuite comme précédemment avec diminution du courant, tout en conservant une valeur inférieure à la pente initiale. Ceci s'apparente à une augmentation des effets de perçage au début de la contrainte [10], mais peut être aussi interprété par une génération de trous par ionisation par impact au voisinage du point de pincement du canal, dans le substrat, entraînant un abaissement de la barrière de potentiel de la jonction source-substrat, et, conséquemment, une injection accrue de la source (effet de transistor bipolaire parasite) $[1,10]$. 

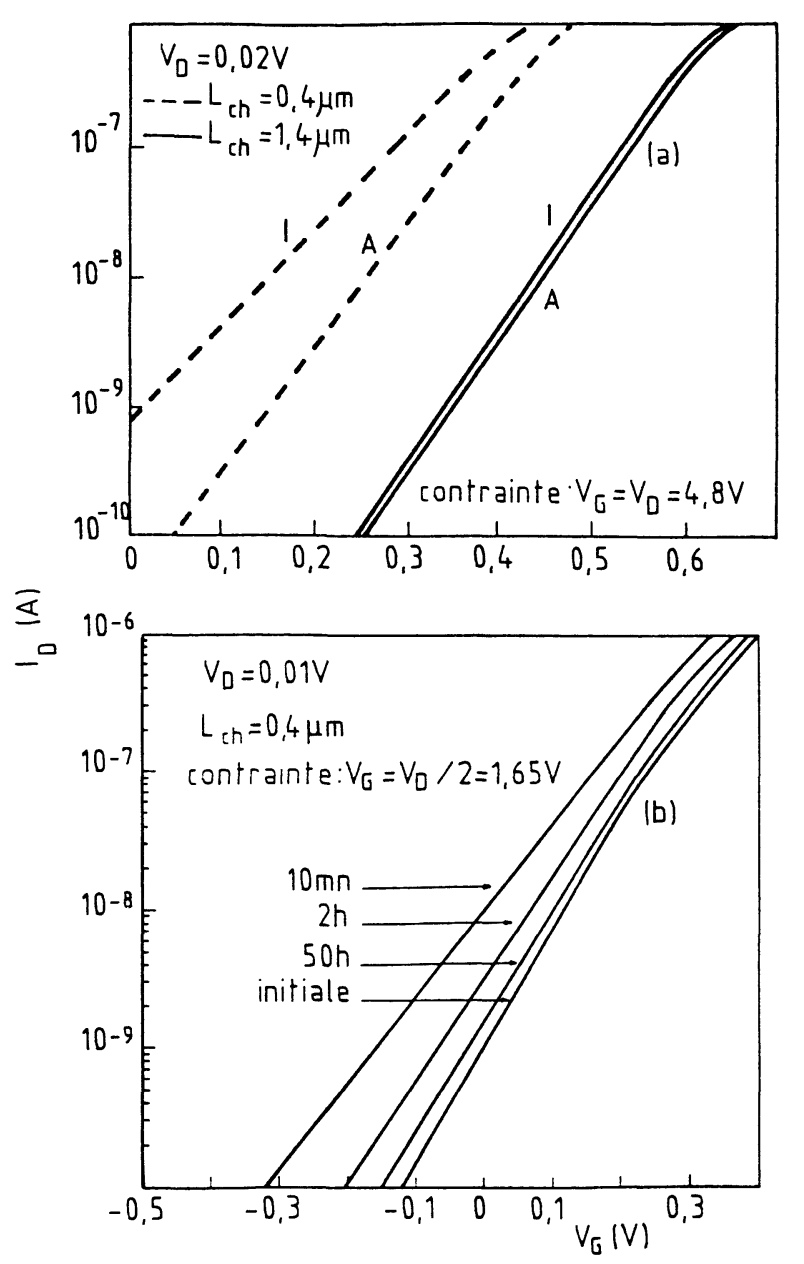

Fig. 4. - a) Caractéristiques de transfert en inversion faible avant (I) et après (A) $50 \mathrm{~h}$ d'une même contrainte $V_{\mathrm{G}}=V_{\mathrm{D}}$ appliquée à un TMOS à canal "long" $\left(L_{\mathrm{ch}}=1,4 \mu \mathrm{m}\right.$, $\longrightarrow$ ) et très court $\left(L_{\mathrm{ch}}=0,4 \mu \mathrm{m},---\right)$. b) Caractéristiques de transfert en inversion faible, initiale, et après trois étapes de vieillissement d'un TMOS à très court canal soumis à la condition $V_{\mathrm{G}}=V_{\mathrm{D}} / 2$.

[a) Transfer characteristics in weak inversion before (I) and after (A) 50 hours of ageing of a « long " channel device $\left(L_{\mathrm{ch}}=1.4 \mu \mathrm{m}, \longrightarrow\right)$ and a very short channel device $\left(L_{\mathrm{ch}}=0.4 \mu \mathrm{m},-\right.$ - - $^{-}$) stressed under the same bias condition. b) Initial transfer characteristics in weak inversion and its evolution after three aging steps of a very short channel device stressed under the bias condition $V_{\mathrm{G}}=V_{\mathrm{D}} / 2$.]

Enfin, nous présentons sur la figure 5 la corrélation entre les dégradations expérimentales du maximum $\mu_{\mathrm{m}}$ de mobilité et celles de la tension de seuil associées $V_{\mathrm{Gm}}$ pour des transistors à canal très court $\mathrm{T}_{1}, \mathrm{~T}_{2}$ et $\mathrm{T}_{3}$. Les points expérimentaux de chaque courbe correspondent à des étapes de vieillissement ayant la même durée. Pour les deux conditions de contrainte $V_{\mathrm{G}}<V_{\mathrm{D}}$ et $V_{\mathrm{G}}=V_{\mathrm{D}}$, les points représentatifs se trouvent sur la même courbe mais avec une amplitude de dégradation finale plus faible pour la condition $V_{\mathrm{G}}<V_{\mathrm{D}}$. La courbe relative à la condition $V_{\mathrm{G}}>V_{\mathrm{D}}$, quant à elle, ne se superpose pas avec les précédentes :

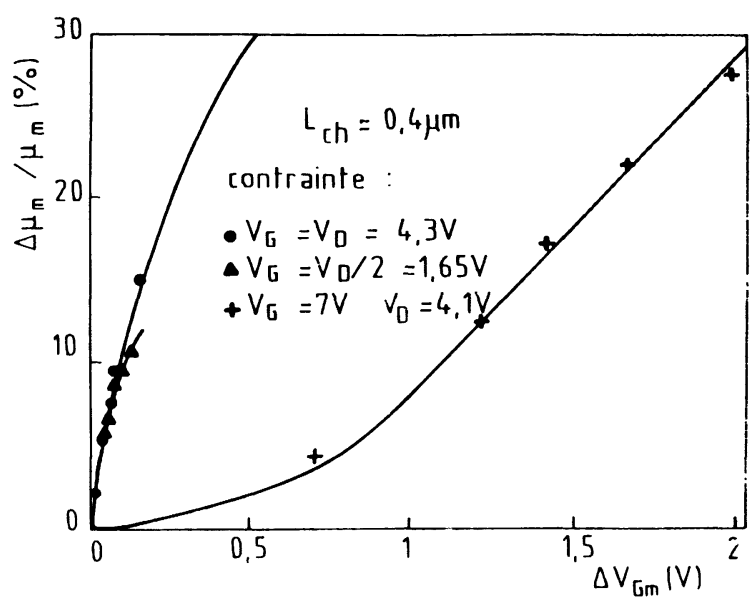

Fig. 5. - Corrélation expérimentale entre la dégradation relative du maximum de mobilité $\mu_{\mathrm{m}}$ et la variation de la tension de seuil de mobilité $V_{\mathrm{Gm}}$ pour un TMOS à canal très court soumis à trois contraintes différentes. Cinq points ont été sélectionnés sur chaque courbe et correspondent aux mêmes durées de vieillissement : $10 \mathrm{~min}, 2 \mathrm{~h}, 5 \mathrm{~h}, 10 \mathrm{~h}$, $25 \mathrm{~h}$.

[Experimental correlation between the relative degradation of the mobility maximum $\mu_{\mathrm{m}}$ and the variation of the mobility threshold voltage $V_{\mathrm{Gm}}$ for a very short channel device stressed under three bias conditions. Five points have been selected on each curve and correspond to the same stress time : $10 \mathrm{~min}, 2 \mathrm{~h}, 5 \mathrm{~h}, 10 \mathrm{~h}, 25 \mathrm{~h}$.]

les variations importantes de $V_{G m}$ témoignent d'une plus grande création d'états d'interface et/ou d'un piégeage important de charges négatives dans l'oxyde, et ceci dès le début de la contrainte.

Les caractéristiques de corrélation entre les dégradations de tension de seuil et de mobilité présentent des résultats analogues dans le cas de transistors de moins courtes longueurs de canal $\left(\mathrm{T}_{4}\right.$ et $\left.\mathrm{T}_{5}\right)$. Elles seront étudiées et modélisées dans le paragraphe suivant.

\section{Modélisation}

Quelques modèles de dégradation de mobilité ont déjà été considérés dans la littérature; ces modèles empiriques font état d'une décroissance hyperbolique du maximum de mobilité avec les valeurs absolues des charges fixes $Q_{\mathrm{f}}$ et des charges d'états d'interface $Q_{\mathrm{ss}}$ créés [12] :

$$
\mu_{\mathrm{m}}=\frac{\mu_{0}}{1+\alpha\left(Q_{\mathrm{SS}}+Q_{\mathrm{f}}\right)} .
$$

Cette décroissance a été expliquée par des collisions avec les centres chargés de l'interface et les phonons. Ce type de modèle a été utilisé par la suite pour corréler les dégradations de transconductance et de tension de seuil à la création d'états d'interface [2]. La diminution relative de transconductance $G_{G}$ est alors proportionnelle à la densité $N_{\mathrm{ss}}$ d'états d'interface 
créés selon la relation :

$$
\frac{\Delta G_{\mathrm{G}}}{G_{\mathrm{G}_{0}}-\Delta G_{\mathrm{G}}}=\alpha N_{\mathrm{SS}} .
$$

Des observations expérimentales, utilisant le pompage de charge avec $V_{\mathrm{G}}<V_{\mathrm{D}}$, ont confirmé cette corrélation entre la dégradation de mobilité et l'augmentation d'états d'interface $[3,5,6]$, lorsque les états créés sont des états accepteurs. Néanmoins, ces mêmes auteurs ont remarqué une absence de corrélation lorsque une contrainte $V_{\mathrm{G}}=V_{\mathrm{D}}$ est appliquée au TMOS, qui engendre des états donneurs par injection d'électrons chauds. C'est dans ce cas que nous avons observé des dégradations maximum et nous ne pourrons pas les interpréter par de tels modèles.

Dans ce travail, les dégradations expérimentales de mobilité se manifestent essentiellement dans la région du maximum de mobilité (moyenne inversion) et en faible inversion. Cela suggère un mécanisme de dégradation selon lequel l'écrantage électrostatique dû à la charge des porteurs de la couche d'inversion atténue en forte inversion les effets qui induisent la réduction de mobilité. Un tel processus complexe de dégradation de mobilité doit reposer sur les effets des fluctuations de potentiel à l'interface et des phénomènes de localisation des porteurs qui en résultent [22-24].

4.1 DESCRIPTION DU MODÈLE UTILISÉ. - Plusieurs modèles de fonctionnement du TMOS enrichi ont été proposés [25-30]. Ces modèles prennent en compte les principaux effets de canaux courts tels que la modulation de la longueur effective du canal par le potentiel de drain, la saturation de vitesse des porteurs, la réduction de mobilité en forte inversion, les courants d'inversion faible, etc.

Bien que ces modèles décrivent avec précision le fonctionnement du TMOS en régime d'inversion forte, ils ne donnent pas de description complète de la conductance et de la transconductance dans toute l'étendue des régimes d'inversion du transistor. Une telle description doit en effet être continue de la faible à la forte inversion et utilise une loi simple de dépendance de la mobilité effective avec la charge dans la couche d'inversion [15] :

$$
\mu_{\text {eff }}=\frac{\mu_{0}}{\left[1+Q_{\mathrm{i}} / Q_{\mathrm{c}}\right]}
$$

où $\mu_{0}$ est une mobilité constante dépendant des mécanismes de collisions, $Q_{\mathrm{c}}$ est une charge critique $\left(Q_{\mathrm{c}} \sim 10^{13} \mathrm{qcm}^{-2}\right)$ à partir de laquelle la mobilité décroît [15], $Q_{\mathrm{i}}$ est la charge dans la couche d'inversion.

Pour un TMOS à canal $\mathrm{n}$ dont le dopage du substrat est $N_{\mathrm{A}}$, les lois de dépendance de $Q_{\mathrm{i}}$ et de $V_{\mathrm{G}}$ avec le potentiel de surface $\Psi_{\mathrm{S}}$ sont données par [31] :

$Q_{\mathrm{i}}=\left[\left[\left(Q_{\mathrm{D}}+Q_{\mathrm{SS}}\right)^{2}+4 q \varepsilon_{\mathrm{Si}} \frac{k T}{q} \frac{n_{\mathrm{i}}^{2}}{N_{\mathrm{A}}} \times\right.\right.$

$$
\begin{array}{r}
\left.\left.\times \exp \left(\frac{q \Psi_{\mathrm{s}}}{k T}\right)\right]^{1 / 2}-\left(Q_{\mathrm{D}}+Q_{\mathrm{SS}}\right)\right] / 2 \\
V_{\mathrm{G}}=V_{\mathrm{FB}}+\Psi_{\mathrm{s}}+\left(Q_{\mathrm{i}}+Q_{\mathrm{D}}+Q_{\mathrm{SS}}\right) / C_{\mathrm{ox}}
\end{array}
$$

avec $q=$ charge absolue de l'électron, $k=$ constante de Boltzman, $\varepsilon_{\mathrm{Si}}=$ permittivité du silicium, $n_{\mathrm{i}}=$ concentration intrinsèque des porteurs, $T=$ température absolue, $V_{\mathrm{FB}}=$ tension de bandes plates et $Q_{\mathrm{D}}$ est la charge absolue de la zone désertée :

$$
Q_{\mathrm{D}}=\left[2 q \varepsilon_{\mathrm{Si}} N_{\mathrm{A}}\left(\Psi_{\mathrm{s}}-k T / q\right)\right]^{1 / 2}
$$

et $Q_{\mathrm{Ss}}$ est la charge absolue des états d'interface mesurée par rapport à la condition de bandes plates :

$$
Q_{\mathrm{SS}}=q N_{\mathrm{SS}} \Psi_{\mathrm{s}}
$$

et $N_{\text {ss }}$ est la densité d'états d'interface supposée constante sur tout le gap. La relation (6) est valable dans tous les régimes d'inversion. En effet :

- dans le régime d'inversion forte, la charge d'inversion $Q_{\mathrm{i}}$ est sensiblement égale à $C_{\mathrm{ox}}\left(V_{\mathrm{G}}-V_{\mathrm{T}}\right)$ ( $V_{\mathrm{T}}=$ tension de seuil de la charge d'inversion) et la loi de mobilité prend la forme de la relation (4) dans laquelle $\theta=C_{\mathrm{ox}} / Q_{\mathrm{c}}$;

- la relation (6) permet d'intégrer également les effets dus aux fluctuations de potentiel et agissant dans les régimes de faible et moyenne inversions. Ces fluctuations de potentiel entraînent un désordre et une localisation des porteurs en bord de bande, ce qui est analytiquement décrit par $[15,23,24]$ :

$$
\mu_{0}=\mu_{00} \exp \left[-\frac{\Gamma}{k T}\right] \text { avec } \Gamma \simeq q \sigma_{\mathrm{s}}
$$

où $\mu_{00}$ est la valeur de la mobilité en l'absence de fluctuations de potentiel, et $\sigma_{\mathrm{s}}$ est l'écart type de la distribution du potentiel de surface relié à l'écart type de la distribution de charge $\sigma_{\mathrm{q}}$ par une relation capacitive de la forme :

$$
\sigma_{\mathrm{s}}=\frac{\sigma_{\mathrm{q}}}{C_{\mathrm{ox}}+C_{\mathrm{D}}+C_{\mathrm{SS}}+C_{\mathrm{i}}}
$$

dans laquelle $C_{\mathrm{D}}, C_{\mathrm{SS}}, C_{\mathrm{i}}$ sont respectivement les capacités de déplétion $\left(C_{\mathrm{D}}=\delta Q_{\mathrm{D}} / \delta \Psi_{\mathrm{s}}\right)$, d'états d'interface $\left(C_{\mathrm{SS}}=q N_{\mathrm{SS}}\right)$, et de charge dans la couche d'inversion $\left(C_{\mathrm{i}}=\delta Q_{\mathrm{i}} / \delta \Psi_{\mathrm{s}}\right)$.

Ainsi les relations (6) à (10) permettent une analyse $\mathrm{du}$ fonctionnement du TMOS en régime ohmique continûment de la faible à la forte inversion et permettent de décrire la mobilité d'effet de champ en fonction de la tension de grille.

Les courbes $\mu_{\mathrm{FE}}\left(V_{\mathrm{G}}\right)$ seront simulées compte tenu des différents paramètres du TMOS extraits des caractéristiques expérimentales d'inversion forte $(\theta$, $\left.R_{\mathrm{SD}}, \mu_{0}\right)[14,16]$ et des caractéristiques expérimentales d'inversion faible $\left(N_{\mathrm{A}}, \Gamma, N_{\mathrm{SS}}, V_{\mathrm{FB}} \ldots\right)$. On considérera que les principaux paramètres affectés par le vieillisse- 
ment seront le taux de fluctuations $\Gamma$, la densité d'états d'interface $N_{\mathrm{SS}}$ et la tension de bandes plates $V_{\mathrm{FB}}$.

4.2 CORRÉlATION ENTRE LES VARIATIONS DE MOBILITÉ ET DE TENSION DE SEUIL. - L'influence des fluctuations de potentiel de surface tant sur les caractéristiques $\mu_{\mathrm{FE}}\left(V_{\mathrm{G}}\right)$ que sur les caractéristiques de transfert $\log I_{\mathrm{D}}\left(V_{\mathrm{G}}\right)$ en inversion faible a été déterminée pour différents taux de désordre $\sigma_{\mathbf{q}}[21]$. La présence de fluctuations de potentiel permet d'expliquer les deux faits expérimentaux suivants : l'importante dégradation de la mobilité $\mu_{\mathrm{FE}}$ autour du maximum $\mu_{\mathrm{m}}$ de mobilité $[2,32]$ et l'augmentation paradoxale de la pente de la caractéristique $\log I_{\mathrm{D}}\left(V_{\mathrm{G}}\right)$ (correspondant à de fortes valeurs de $\sigma_{\mathbf{q}}$ ).

4.2.1 Dégradation du maximum $\mu_{\mathrm{m}}$ de mobilité et des tensions de seuil $V_{\mathrm{Gm}}$ et $V_{\mathrm{Text}}{ }$ - Les figures $6 \mathrm{a}$ et $6 \mathrm{~b}$ présentent, sur les courbes $\mu_{\mathrm{FE}}\left(V_{\mathrm{G}}\right)$ obtenues par le modèle analytique précédent (relátions (3), (6)-(10)), les deux effets caractéristiques dus à la prise en compte de manière indépendante :

- d'une part des fluctuations de potentiel de surface par la variation du paramètre $\sigma_{\mathrm{q}}$ à $N_{\mathrm{ss}}$ constant (Fig. 6a), ce qui diminue $\mu_{\mathrm{m}}$,

- d'autre part de la variation de la densité d'états d'interface $N_{\text {ss }}$ à $\sigma_{\mathrm{q}}$ constant (Fig. 6b), ce qui augmente fortement $V_{\mathrm{Gm}}$.

La figure 7 représente, en traits pleins, ces deux effets pris chacun indépendamment sur les mêmes variables $\mu_{\mathrm{m}}$ et $V_{\mathrm{Gm}}$. On peut remarquer que les variations relatives de $\mu_{\mathrm{m}}$ et absolues de $V_{\mathrm{Gm}}$ sont corrélées par une relation linéaire à faible coefficient lorsque varie le paramètre $N_{\mathrm{ss}}$ et à fort coefficient lorsque varie le paramètre $\sigma_{q}$. On peut donc en conclure que ni les effets liés à $N_{\mathrm{Ss}}$ seul ni ceux liés à $\sigma_{\mathrm{q}}$ seul ne peuvent expliquer à la fois d'importantes variations de $\mu_{\mathrm{m}}$ et de la tension de seuil associée $V_{\mathbf{G m}}$. Ces deux dégradations observées simultanément doivent s'interpréter par une interdépendance entre ces deux phénomènes. En particulier, la dégradation de la transconductance ne peut s'expliquer seulement par une augmentation de la densité d'états d'interface $[2,17,19]$.

De plus, les figures 5 et 7 présentent des similitudes : leur comparaison suggère que, dans le cas d'un TMOS à canal très court $\left(L_{\mathrm{ch}}=0,4 \mu \mathrm{m}\right)$, la dégradation importante du maximum de mobilité $\mu_{\mathrm{m}}$ s'explique par un effet dominant des fluctuations de potentiel lorsque $V_{\mathrm{G}}<V_{\mathrm{D}}$ et $V_{\mathrm{G}}=V_{\mathrm{D}}$ (cas de $\mathrm{T}_{1}$ et $\left.\mathrm{T}_{2}\right)$, tandis que les dégradations des tensions de seuil liées à la création d'états d'interface et/ou de charges fixes piégées dans l'oxyde prennent autant d'importance que ces fluctuations de potentiel lorsque $V_{G}>V_{D}$ (cas de $\mathrm{T}_{3}$ ).

Les fortes valeurs de la dégradation de la mobilité sont donc liées à la présence de fluctuations de potentiel et sont justifiées par le caractère exponentiel de l'atténuation de la mobilité effective avec le désordre (relation (9)). Une simple loi homographique telle que la relation $(5)[2,12]$ ne suffit pas à expliquer de telles

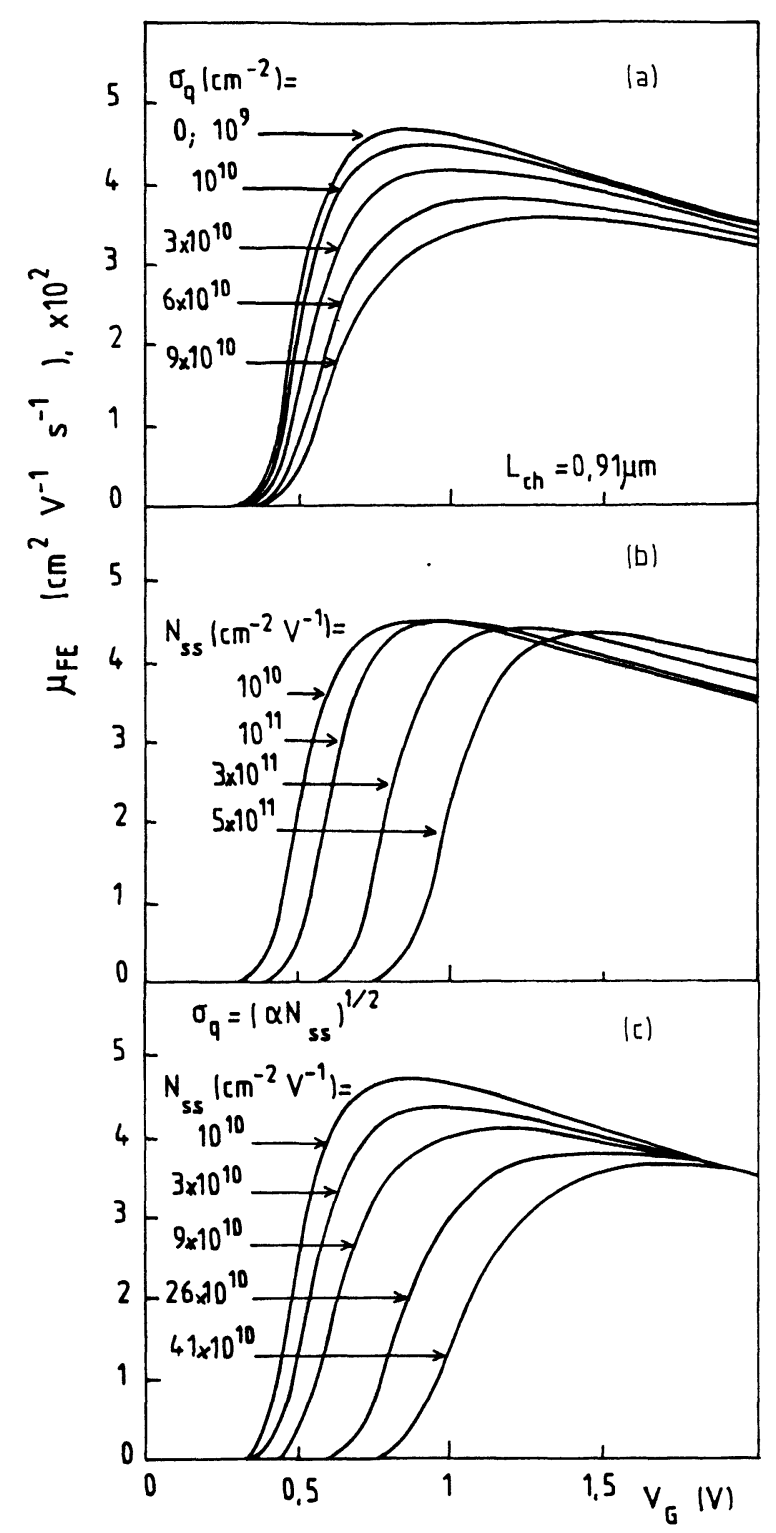

Fig. 6. - Caractéristique $\mu_{\mathrm{FE}}\left(V_{\mathrm{G}}\right)$ calculée selon le modèle avec pour paramètre : a) $\sigma_{\mathrm{q}}\left(N_{\mathrm{Ss}}\right.$ constant $=10^{10} \mathrm{~V}^{-1} \mathrm{~cm}^{-2}$, $\operatorname{voir} \S 4.2 \mathrm{a})$; b) $N_{\mathrm{ss}}\left(\sigma_{\mathrm{q}}\right.$ constant $=10^{10} \mathrm{qcm}^{-2}$, voir $\left.\S 4.2 \mathrm{a}\right)$; c) $N_{\mathrm{ss}}\left(\sigma_{\mathrm{q}}=\left[\alpha N_{\mathrm{ss}}\right]^{1 / 2}\right.$, voir $\left.\S 4.2 \mathrm{~b}\right)$. (Epaisseur d'oxyde de grille $26 \mathrm{~nm} ; \theta=0,05 \mathrm{~V}^{-1} ; R_{\mathrm{SD}}=50 \Omega$ ).

[Characteristics $\mu_{\mathrm{FE}}\left(V_{\mathrm{G}}\right)$ according to our model with : a) $\sigma_{\mathrm{q}}$ as a parameter (constant $N_{\mathrm{ss}}=10^{10} \mathrm{~V}^{-1} \mathrm{~cm}^{-2}$, $\S 4.2 \mathrm{a}$ ); b) $N_{\mathrm{ss}}$ as a parameter (constant $\sigma_{\mathrm{q}}=10^{10} \mathrm{qcm}^{-2}$, $\S 4.2 \mathrm{a})$; c) $N_{\mathrm{ss}}$ as a parameter $\left(\sigma_{\mathrm{q}}=\left[\alpha N_{\mathrm{ss}}\right]^{1 / 2}, \S 4.2 \mathrm{~b}\right)$. (Gate oxide thickness $26 \mathrm{~nm} ; \theta=0.05 \mathrm{~V}^{-1} ; R_{\mathrm{SD}}=50 \Omega$ ).]

dégradations de mobilité pouvant atteindre $30 \%$ de la valeur initiale.

Examinons maintenant les deux effets, ceux des fluctuations de potentiel et ceux des états d'interface toujours considérés séparément, sur la tension de seuil extrapolée $V_{\text {Text }}$ (courbes en traits pleins de la Fig. 8). Il apparaît sur cette figure que l'augmentation de la tension de seuil $\Delta V_{\text {Text }}$ est linéaire avec celle de la densité détats d'interface $N_{\text {ss }}$, mais que l'augmentation 


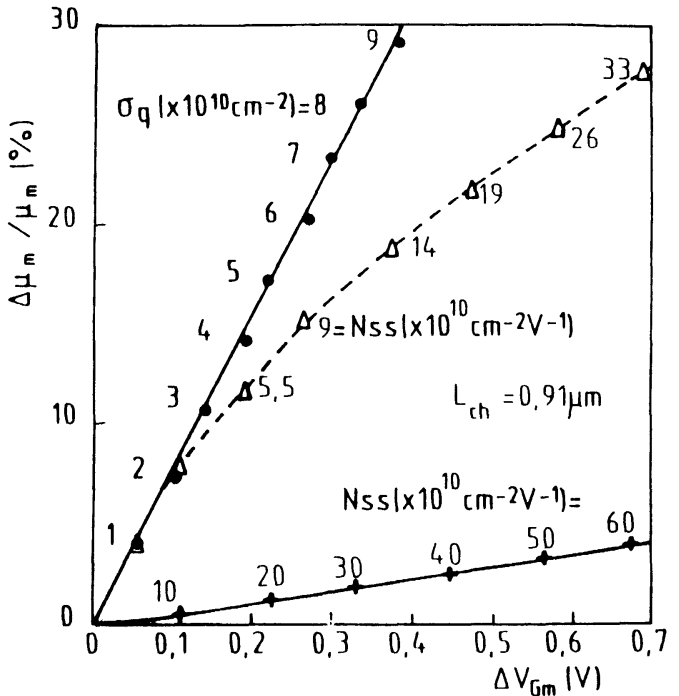

Fig. 7. - Corrélation théorique entre la variation relative du maximum $\mu_{\mathrm{m}}$ de mobilité et la variation de la tension de mobilité $V_{\mathrm{Gm}}$ avec pour paramètre : $\sigma_{\mathrm{q}}\left(N_{\mathrm{ss}}\right.$ constant $=$ $\left.10^{10} \mathrm{~cm}^{-2} \mathrm{~V}^{-1}, \longrightarrow-\right) ; N_{\mathrm{Ss}}\left(\sigma_{\mathrm{q}}\right.$ constant $=10^{10} \mathrm{qcm}^{-2}$, $-+-) ; N_{\mathrm{Ss}}\left(\right.$ relié à $\sigma_{\mathrm{q}}$ par : $\left.\sigma_{\mathrm{q}}=\left(\alpha N_{\mathrm{SS}}\right)^{1 / 2},--\Delta--\right)$ ).

[Theoretical correlation between the relative variation of the mobility maximum $\mu_{\mathrm{m}}$ and absolute variation of the mobility threshold voltage $V_{\mathrm{Gm}}$ with $: \sigma_{\mathrm{q}}$ as a parameter (constant $N_{\mathrm{Ss}}=10^{10} \mathrm{~V}^{-1} \mathrm{~cm}^{-2}, \longrightarrow$ ) $; N_{\mathrm{Ss}}$ as a parameter (constant $\sigma_{\mathrm{q}}=10^{10} \mathrm{qcm}^{-2},-+-$ ); $N_{\mathrm{ss}}$ (correlated with $\sigma_{\mathrm{q}}$ according : $\sigma_{\mathrm{q}}=\left(\alpha N_{\mathrm{SS}}\right)^{1 / 2},--\triangle-$ - $)$.]

des fluctuations $\sigma_{\mathrm{q}}$ entraînent une diminution de $V_{\text {Texr. }}$ Les fluctuations de potentiel, dans un régime où domine la dégradation de mobilité, peuvent donc à elles seules expliquer les variations très faibles de tension de seuil extrapolée $V_{\text {Text }}$ (Fig. 2) sans invoquer de piégeage de charges positives dans l'oxyde. Cette tension de seuil, obtenue par extrapolation de la courbe $G_{\mathrm{D}}\left(V_{\mathrm{G}}\right)$ au point d'inflexion vers une conductance nulle, peut être reliée à la conductance $G_{\mathrm{Dm}}$, à la transconductance normalisée aux faibles tensions de drain $G_{\mathrm{Gdm}}\left(G_{\mathrm{Gd}}=\delta G_{\mathrm{D}} / \delta V_{\mathrm{G}}\right)$, et à la tension de seuil de mobilité $V_{\mathrm{Gm}}$, toutes trois prises au maximum de mobilité :

$$
V_{\mathrm{Text}}=V_{\mathrm{Gm}}-\frac{G_{\mathrm{Dm}}}{G_{\mathrm{Gdm}}}
$$

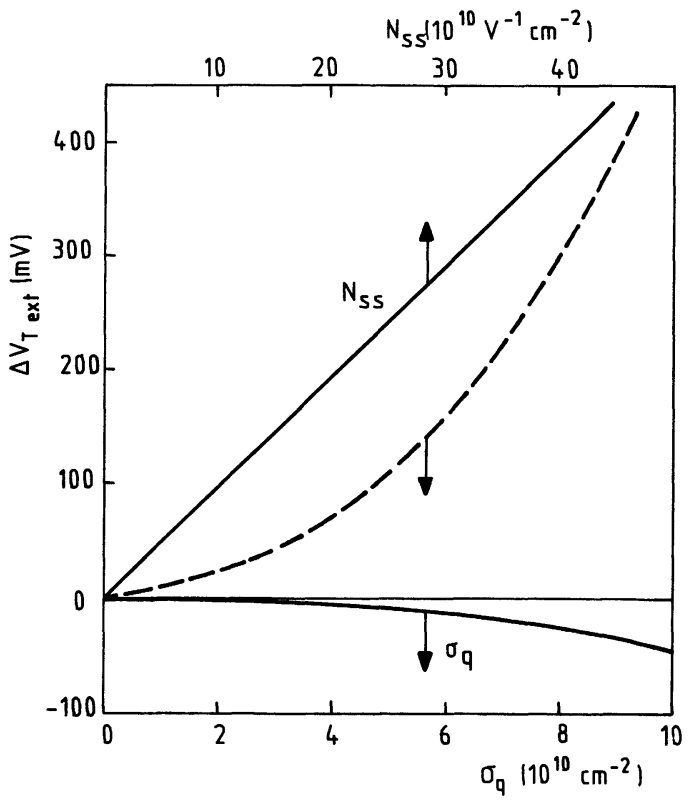

Fig. 8. - Variation théorique de la tension de seuil extrapolée $V_{\text {Text }}$ avec : $\sigma_{\mathrm{q}}\left(N_{\mathrm{ss}}\right.$ constant $\left.=10^{10} \mathrm{~cm}^{-2} \mathrm{~V}^{-1}\right)$ et $N_{\mathrm{ss}}\left(\sigma_{\mathrm{q}}\right.$ constant $\left.=10^{10} \mathrm{qcm}^{-2}\right)$ en traits pleins : $\sigma_{\mathrm{q}}$ relié à $N_{\mathrm{ss}}$ par : $\sigma_{\mathrm{q}}=\left(\alpha N_{\mathrm{SS}}\right)^{1 / 2}$ en traits pointillés.

[Theoretical variation of the extrapolated threshold voltage $V_{\text {Text }}$ with : $\sigma_{\mathrm{q}}\left(\right.$ constant $\left.N_{\mathrm{SS}}=10^{10} \mathrm{~cm}^{-2} \mathrm{~V}^{-1}\right)$ and $N_{\text {SS }}$ (constant $\sigma_{\mathrm{q}}=10^{10} \mathrm{qcm}^{-2}$ ) in solid line; $\sigma_{\mathrm{q}}$ correlated with $N_{\mathrm{ss}}$ according to : $\sigma_{\mathrm{q}}=\left(\alpha N_{\mathrm{sS}}\right)^{1 / 2}$ in dotted line.]

Finalement, on peut corréler les variations différentielles (au premier ordre) du maximum $\mu_{\mathrm{m}}$ de mobilité, celles de la tension de seuil associée $V_{\mathrm{Gm}}$, et celles de la tension de seuil extrapolée $V_{\text {Text }}$ entre elles par [33] :

$$
\delta V_{\mathrm{Text}}=\delta V_{\mathrm{Gm}}+\frac{G_{\mathrm{Dm}}}{G_{\mathrm{Gdm}}} \frac{\delta \mu_{\mathrm{m}}}{\mu_{\mathrm{m}}} .
$$

Cette relation permet, par exemple, d'analyser la corrélation entre les variations expérimentales des différents paramètres d'un transistor $\left(T_{5}\right)$, après vieillissement (voir tableau II). Ainsi, deux régimes peuvent être observés :

- lorsque les fluctuations de potentiel prédominent, entraînant une forte diminution de mobilité $\left(\delta \mu_{\mathrm{m}} / \mu_{\mathrm{m}}<0\right)$ et une faible augmentation de la tension

Tableau II. - Correlation entre les variations des paramètres $V_{\text {Text }}, V_{\mathrm{Gm}}$, et $\mu_{\mathrm{m}}$, obtenue expérimentalement et calculée d'après la relation (12), correspondant à deux valeurs de $N_{\text {ss. }}$.

[Correlation between the variations of $V_{\text {Text }}, V_{\mathrm{Gm}}$, and $\mu_{\mathrm{m}}$, in practice and according to relation (12) corresponding to two $N_{\mathrm{SS}}$ values.]

\begin{tabular}{ccccccr} 
Etape & $\Delta \mu_{\mathrm{m}} / \mu_{\mathrm{m}}$ & $\begin{array}{c}\Delta V_{\mathrm{Gm}} \\
(\mathrm{V})\end{array}$ & $\begin{array}{c}G_{\mathrm{Dm}} / G_{\mathrm{Gdm}} \\
(\mathrm{V})\end{array}$ & $\begin{array}{c}\Delta V_{\text {Text }}(\text { Théor. }) \\
(\mathrm{V})\end{array}$ & $\begin{array}{c}\Delta V_{\text {Text }}(\text { Exp. }) \\
(\mathrm{V})\end{array}$ & $\begin{array}{c}N_{\mathrm{Ss}} \\
\left(\mathrm{V}^{-1} . \mathrm{cm}^{-2}\right)\end{array}$ \\
\hline $10 \mathrm{~h}$ & $-0,14$ & 0,13 & 0,39 & $+0,090$ & $-0,008$ & $4 \times 10^{10}$ \\
$70 \mathrm{~h}$ & $-0,20$ & 1 & 0,86 & $+0,820$ & $+0,920$ & $26 \times 10^{10}$
\end{tabular}


de seuil associée $\delta V_{\mathrm{Gm}}\left(\delta V_{\mathrm{Gm}}>0\right)$ (ces deux variations sont reliées linéairement sur la Fig. 7), la variation de tension de seuil extrapolée $\delta V_{\text {Text }}$ est très faible; - lorsque prédomine la création d'états d'interface et/ou l'injection de charges négatives dans l'oxyde, on observe une grande variation de tension de seuil de mobilité $\delta V_{\mathrm{Gm}}$ tandis que la variation relative du maximum de mobilité peut saturer $\left(\delta \mu_{\mathrm{m}} / \mu_{\mathrm{m}}=0\right)$. Dans ce cas, le second terme de la relation (12) pouvant être négligé, la variation de tension de seuil extrapolée $V_{\text {Text }}$ est nettement positive et se rapproche de celle de $V_{\mathrm{Gm}}: \delta V_{\mathrm{Gm}} \# \delta V_{\mathrm{Text}}$. Entre ces deux régimes, la variation de la tension de seuil extrapolée peut changer de signe (voir Fig. 2b).

De plus, différents facteurs parasites peuvent également modifier la tension de seuil extrapolée : la résistance série $R_{\mathrm{SD}}$, le coefficient $\theta$ de réduction de mobilité [16]. Cela montre pourquoi cette tension de seuil $V_{\text {Text }}$ ne peut pas être considérée comme un paramètre fondamental du TMOS comme l'a déjà mentionné Brews [34].

Nous avons déjà remarqué (Figs. 6 et 7) que la dégradation de la tension de seuil $V_{\mathrm{Gm}}$ s'interprète notamment par une augmentation de la densité des états d'interface (à laquelle peut s'ajouter une augmentation des charges fixes), et que la dégradation du maximum de mobilité $\mu_{\mathrm{m}}$ s'interprète, quant à elle, principalement par une augmentation des fluctuations de charges dues à la présence d'états chargés à l'interface $\mathrm{Si} / \mathrm{SiO}_{2}$. Or, les dégradations de ces deux paramètres $V_{\mathrm{Gm}}$ et $\mu_{\mathrm{m}}$ se trouvent simplement liées par la relation (12). Il reste donc à corréler les fluctuations de charge à l'augmentation de la densité d'états d'interface, ainsi un seul paramètre, $N_{\mathrm{SS}}$, permettra de contrôler les taux de dégradations. Un tel modèle de dégradation est envisagé au paragraphe suivant.

4.2.2 Modèle analytique de dégradation. - Généralement, les modèles courants $[2,5,6,12,35-37]$ considèrent que les paramètres du TMOS évoluent selon les relations :

$$
-\frac{\Delta S}{S}=-\frac{\Delta G_{\mathrm{G}}}{G_{\mathrm{G}}}=\frac{\Delta V_{\mathrm{T}}}{V_{\mathrm{T}}}=\frac{\Delta N_{\mathrm{SS}}}{N_{\mathrm{SS}}} .
$$

Ces modèles sont inadaptés à l'interprétation de nos résultats car ils ne tiennent pas compte des fluctuations de charge responsables des dégradations de mobilité (Fig. 6a) et de changements particuliers de pente de la caractéristique de transfert en inversion faible (Fig. 4).

Il existe une relation non linéaire après plusieurs heures de contraintes entre les dégradations du maximum de mobilité $\Delta \mu_{\mathrm{m}} / \mu_{\mathrm{m}}$ et celles de la tension de seuil associée $\Delta V_{\mathrm{Gm}}$ (voir Fig. 5 expérimentale) : cela suggère que l'origine des fluctuations de potentiel (ou de charges) est étroitement reliée à la création de charges d'interface. En effet, on considère en général que la distribution de charges d'interface est poissonienne, de sorte que l'écart type $\sigma_{\mathrm{q}}$ des fluctuations de charges d'une part, et la somme des charges des états d'interfaces $Q_{\mathrm{ss}}$ et des charges fixes $Q_{\mathrm{f}}$ qui engendrent ces fluctuations d'autre part, sont reliées par une relation quadratique [22] :

$$
\sigma_{\mathrm{q}}=\left[q \frac{\left|\mathrm{Q}_{\mathrm{SS}}\right|+\left|\mathrm{Q}_{\mathrm{f}}\right|}{4 \Pi \lambda^{2}}\right]^{1 / 2}
$$

où $\lambda$ est la longueur caractéristique de la distribution des charges à l'interface. Par suite, on négligera l'influence des charges fixes dans l'oxyde $Q_{\mathrm{f}}$ sur l'écart type des fluctuations $\sigma_{\mathrm{q}}$ et conséquemment sur la dégradation de mobilité, de sorte que, pour interpréter les dégradations observées, on utilisera cette relation sous une forme réduite :

$$
\sigma_{\mathrm{q}}=\left[\alpha N_{\mathrm{Ss}}\right]^{1 / 2}
$$

Dans notre cas, le coefficient d'ajustement $\alpha$ avoisine la valeur $2 \times 10^{10} q^{2} \mathrm{eV} / \mathrm{cm}^{2}$. Cela correspond à une longueur caractéristique $\lambda$ de $30 \mathrm{~nm}$ pour la distribution des charges en surface.

A partir de ce modèle (Rel. (15)), nous avons obtenu les courbes théoriques de la figure $6 \mathrm{c}$ et des figures 7 et 8 (courbe en pointillés) qui reproduisent bien les résultats expérimentaux. Par ailleurs, nous avons étudié expérimentalement différentes conditions de contrainte appliquées aux TMOS. Dans le cas de $\mathrm{T}_{2}, \mathrm{~T}_{4}$ et $\mathrm{T}_{5}$, nous avons observé une variation négligeable du potentiel de bandes plates $V_{\mathrm{FB}}$ après le début de la contrainte ; ceci justifie l'emploi de la relation (14) sous sa forme réduite (15). De plus, on notera que la relation (15) fait intervenir la valeur absolue des charges injectées dans l'oxyde tandis que le potentiel de bandes plates en tient compte par leur somme algébrique; ceci peut expliquer qu'une variation importante de $\sigma_{\mathrm{q}}$ et donc de mobilité soit observée, tout en ayant une variation faible de $V_{\mathrm{FB}}$ et des tensions de seuil. Mais il peut arriver, dans le cas de $\mathrm{T}_{3}$, que l'on observe une variation importante de $V_{\text {FB }}$ après le début de la contrainte (ce qui explique les fortes variations de tensions de seuil apparaissant sur la Fig. 1b); de plus, des variations négatives de la tension de seuil extrapolée peuvent être observées (cas de $T_{1}$ et de $T_{5}$ ) en début de contrainte : la relation réduite (15) ne suffit plus alors tout à fait pour décrire ces phénomènes, elle ne rend pas bien compte de toutes les variations de $V_{\text {Text }}$.

La figure 9 montre les courbes de corrélation entre les variations relatives de mobilité et les variations de la tension de seuil associée : sur cette figure se superposent les courbes obtenues expérimentalement et celle obtenue par le modèle étudié ici (paramétrée selon la densité d'états d'interface créés $N_{\text {ss }}$ reliée à $\sigma_{\mathbf{q}}$ par la relation (15)) pour un transistor de longueur de canal la moins courte $\left(L_{\mathrm{ch}}=1,4 \mu \mathrm{m}\right)$ soumis à deux contraintes différentes. On peut noter une bonne concordance entre le modèle et l'expérience pour des durées relativement courtes de contrainte. Cependant un désaccord existe pour les grandes durées dû pro- 


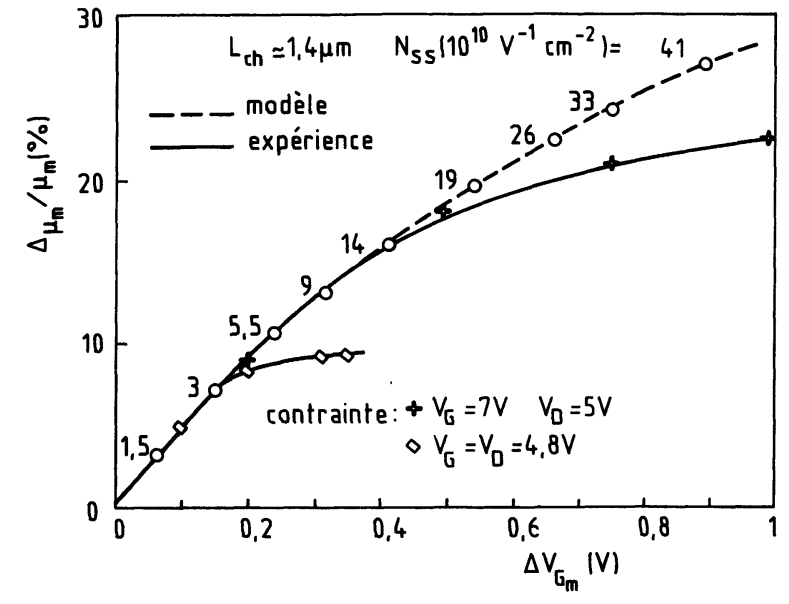

Fig. 9. - Corrélation entre la variation relative du maximum de mobilité $\mu_{\mathrm{m}}$ et la variation de tension de seuil de mobilité $V_{\mathrm{Gm}}$ selon le modèle (-- -) et l'expérience (pour un TMOS à canal « long " soumis à deux contraintes différentes ; 4 points ont été portés sur chaque courbe expérimentale correspondant aux mêmes durées de contrainte : $2 \mathrm{~h}, 10 \mathrm{~h}, 50 \mathrm{~h}, 70 \mathrm{~h}$. La courbe théorique est paramétrée selon $N_{\mathrm{Ss}}$ relié à $\sigma_{\mathrm{q}}$ par : $\sigma_{\mathrm{q}}=\left(\alpha N_{\mathrm{SS}}\right)^{1 / 2}$.

[Correlation between the relative variation of the mobility maximum $\mu_{\mathrm{m}}$ and the absolute variation of the mobility threshold voltage $V_{\mathrm{Gm}}$ according to our model (- -) and and in practice (-) for a "long» channel device stressed under two different bias conditions; four points have been selected on each experimental curve corresponding to the same stress time : $2 \mathrm{~h}, 10 \mathrm{~h}, 50 \mathrm{~h}, 70 \mathrm{~h} . N_{\mathrm{ss}}$ is a further parameter of the theoretical curve and is correlated with $\sigma_{\mathrm{q}}$ according to : $\sigma_{\mathrm{q}}=\left(\alpha N_{\mathrm{SS}}\right)^{1 / 2}$.]

\section{bablement à :}

- la non-prise en compte du rôle de la polarisation de grille $V_{\mathrm{G}}$ appliquée lors de la contrainte sur l'amplitude des dégradations; le modèle donne une seule courbe quelle que soit cette polarisation de grille;

- la carence du modèle reposant sur la relation réduite (15) qui ne permet pas de justifier la saturation observée expérimentalement dans la variation de mobilité après plusieurs heures de contrainte.

Toutefois, en fin de contrainte, des variations plus importantes de tensions de seuil sont observées sur ces figures. Ceci suggère des variations du potentiel de bandes plates $V_{\mathrm{FB}}$ plus importantes en fin de vieillissement, et que nous n'avons pas incluses dans notre modèle (relation réduite (15)). Il est donc possible que les dégradations observées soient le résultat de l'injection de charges positives après le début de contrainte suivie par celle de charges négatives qui viendraient se fixer sur les centres positifs déjà créés [7, 43-45]; cette dernière dominerait en fin de contrainte.

La figure 10 montre pour une même polarisation appliquée mais pour deux longueurs de canaux différentes $\left(\mathrm{T}_{2}\right.$ et $\left.\mathrm{T}_{4}\right)$ la corrélation entre $\Delta \mu_{\mathrm{m}} / \mu_{\mathrm{m}}$ et $\Delta V_{\mathrm{Gm}}$

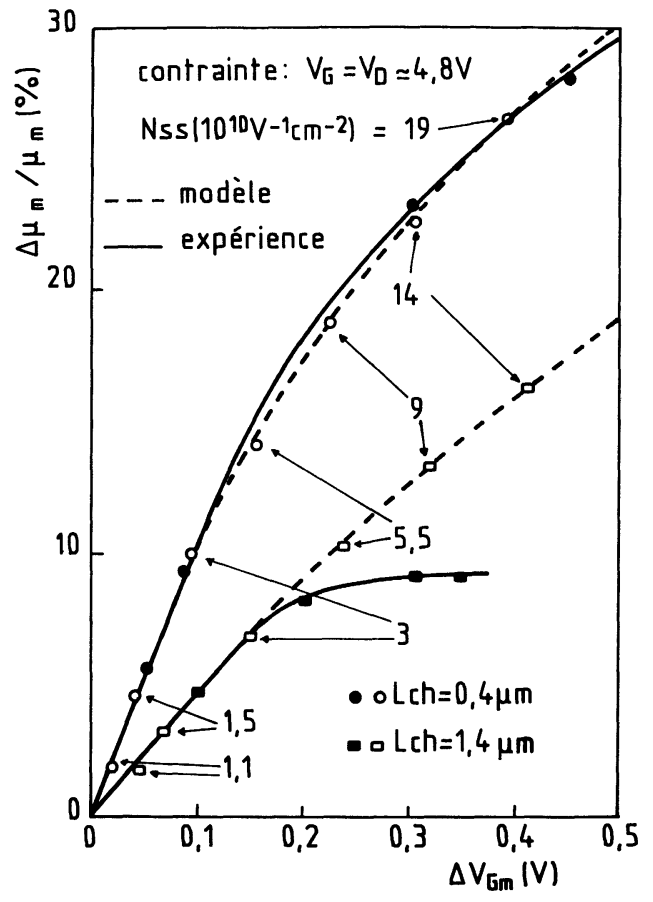

Fig. 10. - Correlation entre les variations du maximum de mobilité $\mu_{\mathrm{m}}$ et la variation de la tension de seuil de mobilité $V_{\mathrm{Gm}}$ selon le modèle (- - -) et l'expérience (-) : rôle de la longueur du canal, pour une même contrainte appliquée; quatre points ont été portés sur chaque courbe expérimentale correspondant aux mêmes durées de contraintes : $2 \mathrm{~h}, 10 \mathrm{~h}, 50 \mathrm{~h}, 70 \mathrm{~h}$. La courbe théorique est paramétrée selon $N_{\mathrm{SS}}$ relié à $\sigma_{\mathrm{q}}$ par : $\sigma_{\mathrm{q}}=\left(\alpha N_{\mathrm{SS}}\right)^{1 / 2}$.

[Correlation between the degradation of the mobility maximum $\mu_{\mathrm{m}}$ and the mobility threshold voltage $V_{\mathrm{Gm}}$ according to our model (- -) and in practice (- $\longrightarrow$ ): influence of the channel length of the MOST stressed under the same bias condition. Four points have been selected on each experimental curve corresponding to the same stress time : $2 \mathrm{~h}, 10 \mathrm{~h}, 50 \mathrm{~h}, 70 \mathrm{~h} . N_{\mathrm{ss}}$ is a further parameter of the theoretical curve and is correlated with $\sigma_{\mathrm{q}}$ according to : $\sigma_{\mathrm{q}}=\left(\alpha N_{\mathrm{SS}}\right)^{1 / 2}$.]

selon l'expérience et selon le modèle étudié ici. Le modèle de dégradation s'applique bien au canal le plus court car aucune saturation de mobilité n'est observée, et au canal le plus long dans le début de la contrainte.

Il est bon de remarquer, d'après les figures 9 et 10 , que la pente à l'origine de ces courbes est caractéristique d'une longueur donnée quelle que soit la contrainte appliquée. Cette dépendance avec la longueur, identique entre le modèle et l'expérience, s'explique par une relation simple reliant $\mu_{\mathrm{m}}$ et $L_{\mathrm{ch}}^{-1}$ via la résistance série $R_{\mathrm{SD}}[16]$ :

$$
\mu_{\mathrm{m}} \simeq \mu_{\mathrm{m}}^{0}\left(1-2 R_{\mathrm{SD}} G_{\mathrm{D}}^{0}\right)
$$

dans laquelle $\mu_{\mathrm{m}}^{0}=$ mobilité intrinsèque, et $G_{\mathrm{D}}^{0}=$ conductance du canal lorsque $R_{\mathrm{SD}}=0$ (variant comme $\left.L_{\mathrm{ch}}^{-1}\right)$. 


\section{Discussions.}

Les dégradations de tensions de seuil et de mobilité présentées ici sont plus importantes, au bout de longues durées, pour les tensions de grille appliquées les plus fortes $\left(V_{\mathrm{G}}>V_{\mathrm{D}}\right.$, où $\left.V_{\mathrm{D}} \simeq V_{\mathrm{a}}\right)$. Le choix du rapport $K=V_{\mathrm{D}} / V_{\mathrm{G}}$ lors de la contrainte commande la nature du processus de dégradation.

La littérature offre des avis controversés sur le choix de $K$ correspondant à une dégradation maximum. Tandis que beaucoup d'auteurs $[1,17,32]$ se placent dans les conditions de maximum de courant de substrat $(K>1)$ pour obtenir une dégradation accélérée des paramètres, il a été prouvé que ces dégradations à taux d'injection constant dans l'oxyde sont minimales pour $K=1$ et augmentent pour $K<1$ $\left(V_{\mathrm{G}}>V_{\mathrm{D}}\right)$ [18], comme dans nos résultats.

Cette contradiction apparente a déjà été expliquée par une absence de corrélation entre l'injection d'électrons chauds dans l'oxyde (évaluée par la mesure des courants de grille) et le taux de dégradation des paramètres [18].

Les différences dans les résultats sont liées à la différence dans la nature des porteurs chauds piégés et à la localisation de la zone d'injection, donc à la distribution du champ électrique le long du canal :

- dans le cas d'un TMOS long, la condition $V_{\mathrm{G}}>V_{\mathrm{D}}(K<1)$ correspond à une injection d'électrons chauds du canal s'étendant sur une large zone près du drain : le champ transversal est homogène sur tout le canal et le champ latéral augmente continûment dans le canal de la source au drain.

- la condition $V_{\mathrm{G}}<V_{\mathrm{D}}(K>1)$ correspond à une injection de porteurs chauds, trous et électrons avalancheux, du substrat et/ou du canal et a lieu de part et d'autre du point de pincement, près du drain, là où le champ latéral est très grand tandis que le champ transversal s'inverse. La condition de maximum de courant de substrat est envisagée, dans ce cas, comme une condition de dégradation importante par certains auteurs car elle correspond à une génération maximum de trous [6]. Mais rappelons que le rôle des trous dans la dégradation des paramètres (mobilité, tension de seuil) reste encore contesté $[2,36]$. Signalons dans ce dernier cas $(K>1)$ l'importance de la tension drain-source $V_{\mathrm{DS}}$ qui, au-delà de la valeur $V_{\mathrm{a}}$, pousse le transistor dans un régime de fonctionnement non autorisé conduisant à une dégradation accrue des paramètres [18]. Cette limite n'a peut-être pas été soigneusement observée dans la littérature. En conclusion, le fait que le champ latéral soit plus grand dans la partie active du canal pour $K>1$ expliquerait que certains résultats publiés [37] s'opposent à ceux présentés ici.

Dans le cas de TMOS très courts étudiés ici, des considérations supplémentaires doivent être prises en compte. Ces TMOS sont tous soumis à des champs latéraux importants, même lorsqu'ils sont polarisés selon la condition $V_{\mathrm{G}}>V_{\mathrm{D}}(K<1)$. La tension de drain $V_{\mathrm{D}}$ appliquée très voisine de $V_{\mathrm{a}}\left(V_{\mathrm{D}}<V_{\mathrm{a}}\right)$ permet un régime de saturation élevé sans pour autant dépasser le point critique $V_{\mathrm{D}}=V_{\mathrm{a}}$ déjà signalé. Ce régime de saturation a lieu, pour ces TMOS ultracourts, bien avant que le canal soit pincé : la tension de drain $V_{\text {Dsat }}$ nécessaire pour obtenir le début de ce régime de saturation est en effet bien inférieure à la valeur $V_{\mathrm{G}}-V_{\mathrm{T}}$. Ceci suggère un effet marqué de saturation de vitesse des porteurs plutôt que l'effet dû à l'égalité des potentiels $V_{\mathrm{G}}$ et $V_{\mathrm{D}}$ [38]. Pour rendre compte de ce phénomène nous avons représenté sur la figure 11 la transconductance en régime de saturation pour les TMOS étudiés ici. Cette figure montre les variations de la transconductance, normalisée selon les dimensions $\left(G_{\mathrm{Gsat}} L_{\mathrm{ch}} / W\right)$, en fonction de la tension de grille $V_{\mathrm{G}}$, pour des tensions de drain appliquées $V_{\mathrm{D}}$ supérieures à la tension de saturation $V_{\text {Dsat }}$. La transconductance en saturation est alors donnée par [39] :

$$
G_{\mathrm{Gsat}} \frac{L_{\mathrm{ch}}}{W}=A v_{\mathrm{sat}} L_{\mathrm{ch}}
$$

où $v_{\text {sat }}$ est la vitesse de saturation des porteurs et $A$ est une constante proche de 1 dans le cas de transistors à très courts canaux [39].

On voit d'après cette figure qu'une saturation précoce intervient dans la vitesse des porteurs, et pour des tensions de grille d'autant plus faibles que la longueur du canal est courte [39]. Lorsque la longueur du canal est réduite, la vitesse des porteurs atteint la valeur limite même de la source et donc tout le long du canal $[36,39]$. Il est probable, dans le cas des vieillissements présentés ici, qu'une forte proportion d'électrons du canal soit en régime de saturation de vitesse; ce phénomène favorise un accroissement du champ latéral, et donc également une injection accrue de porteurs. Enfin, l'injection d'électrons dans l'oxyde est favorisée par de grands champs transversaux;

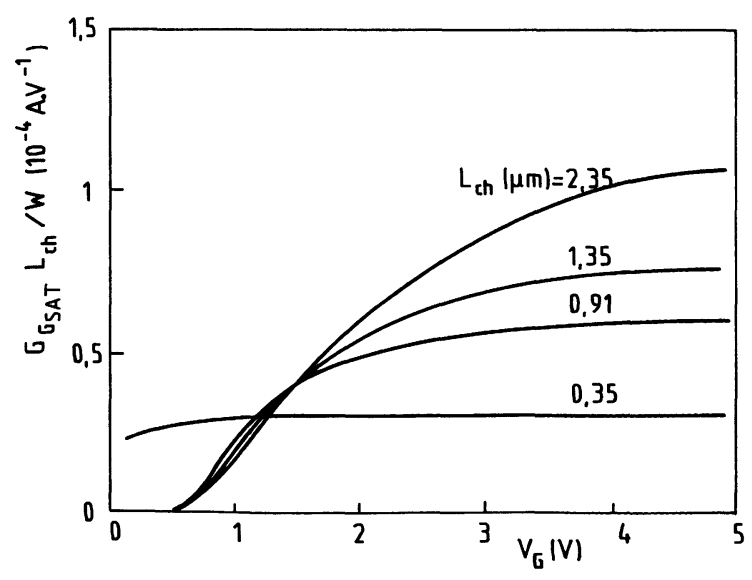

Fig. 11. - Variation expérimentale de la transconductance normalisée en régime de saturation, pour différentes longueurs de canal, avec la tension de grille.

[Experimental variations of the normalized transconductance in the saturation region with gate voltage, with channel length as a further parameter.] 
ces deux dernières remarques permettent de comprendre pourquoi la condition $V_{\mathrm{G}}>V_{\mathrm{D}}(K<1)$ a produit une dégradation accrue pour les transistors étudiés ici.

L'augmentation de dégradation avec la tension de grille appliquée peut s'expliquer en considérant les mécanismes d'injection d'électrons chauds dans la grille, responsables de la dégradation des TMOS. L'étude des caractéristiques des courants de grille et de substrat peut permettre de comprendre ces mécanismes car ils leur sont étroitement corrélés. L'examen des courants de substrat montre, sur la figure 12 :

- pour les canaux les plus longs $\left(L_{\mathrm{ch}}=2,4 \mu \mathrm{m}\right)$ une rapide décroissance du courant de substrat $I_{\text {sub }}$ lorsque la tension de grille augmente au-delà de $2 \mathrm{~V}$;

- pour les canaux assez courts $\left(L_{\mathrm{ch}}=1 \mu \mathrm{m}\right)$ un maximum très large pour les tensions de drain les plus élevées, $V_{\mathrm{D}}>4 \mathrm{~V}$ (la tension de drain appliquée est de l'ordre de $5 \mathrm{~V}$ dans toutes nos expériences), et une décroissance très lente lorsque $V_{\mathrm{G}}$ augmente ;

- pour les transistors à canal très court $\left(L_{\mathrm{ch}}=\right.$ $0,3 \mu \mathrm{m})$, un très large plateau du courant de substrat, sur toute l'étendue des tensions de grille considérée.

Ces phénomènes ont été reliés à des phénomènes d'ionisation par impact qui ont été modélisés à deux dimensions [40]; nos observations expérimentales précédentes, pour $V_{\mathrm{G}}>V_{\mathrm{D}}$, s'expliqueraient par [40] :

- l'accroissement du courant drain-source avec $V_{\mathrm{G}}$ et par une concentration élevée des électrons près du drain, après le point de pincement, ce qui n'est pas observé dans le cas de canaux plus longs à cause de l'inversion du champ transversal dans cette zone;

- un effet de saturation de vitesse des porteurs déjà évoqué ;

- la géométrie des lignes de champ dans la zone désertée du substrat : ces lignes de champ, au lieu de s'écarter de la surface vers le substrat (cas de canaux longs), restent parallèles à la surface (cas de canaux courts) [40]. Le champ électrique latéral déjà élevé dans les très courts canaux entraîne, selon ces considérations, un phénomène d'ionisation par impact accru créant de surcroît des paires électrons-trous plus près de la surface et de l'oxyde dans laquelle ces porteurs pourront être injectés.

Quant aux courants de grille, un comportement analogue a déjà été observé [41] : pour de très courtes longueurs de canaux, la caractéristique $I_{\mathrm{G}}\left(V_{\mathrm{G}}\right)$ exhibe un maximum très plat, et une très faible décroissance de $I_{\mathrm{G}}$ avec $V_{\mathrm{G}}$ pour $V_{\mathrm{G}}>V_{\mathrm{D}}$; dans cette zone de fonctionnement $I_{\mathrm{G}}$ et $I_{\text {sub }}$ ont d'ailleurs été corrélés [41] : ce modèle de corrélation peut rendre compte de l'analogie de comportement (faible diminution de ces courants) pour les fortes tensions de grille.

Toutes ces considérations justifient en grande partie pourquoi la dégradation induite par l'injection dans l'oxyde soit favorisée dans la condition $V_{\mathrm{G}}>V_{\mathrm{D}}$. De récentes mesures de bruit opérées sur les mêmes types de transistors confirment ce résultat [42].

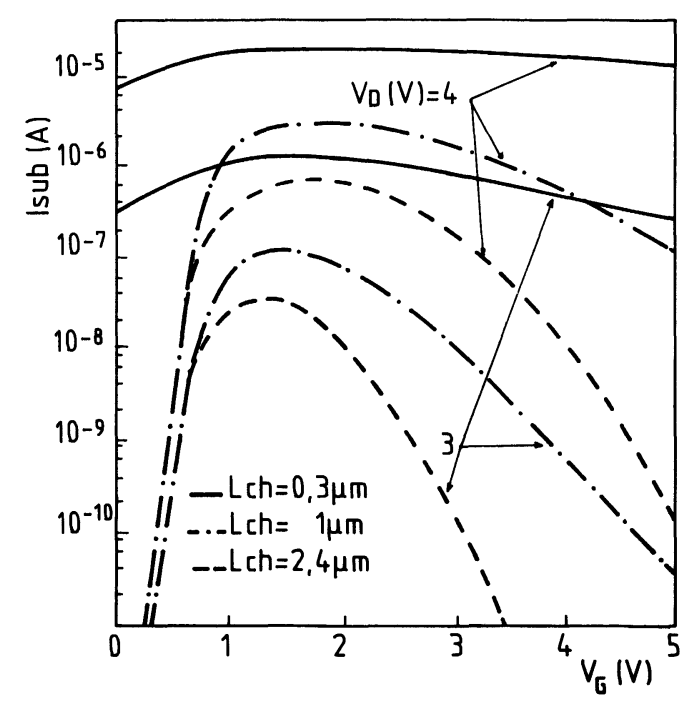

Fig. 12. - Variation expérimentale du courant de substrat avec la tension de grille pour deux différentes tensions de drain, la longueur du canal étant prise comme paramètre.

[Experimental variations of the substrate current with gate voltage for two different drain voltages, with channel length as a further parameter.]

\section{Conclusion.}

La caractéristique $\mu_{\mathrm{FE}}\left(V_{\mathrm{G}}\right)$ apparaît comme étant la mieux adaptée à l'étude expérimentale du vieillissement en régime ohmique : elle renseigne sur l'évolution de la tension de seuil de mobilité et du maximum de mobilité. Nous proposons ici une relation analytique permettant de décrire cette mobilité d'effet de champ dans tous les régimes d'inversion. Celle-ci repose sur la prise en compte des fluctuations de potentiel à l'interface ce qui nous permet de modéliser la dégradation observée au voisinage du maximum de mobilité de la caractéristique $\mu_{\mathrm{FE}}\left(V_{\mathrm{G}}\right)$ et d'expliquer aussi le changement paradoxal de la pente de la caractéristique de transfert en inversion faible. Ces fluctuations sont engendrées par les charges de surface et les charges fixes et sont plus importantes pour de faibles densités de porteurs. Elles agissent donc totalement en régimes d'inversion faible et de transition, précisémment là où ont été observées les plus fortes dégradations. Enfin, nous avons corrélé les dégradations du maximum de mobilité et des tensions de seuil entre elles, et les avons reliées, grâce à un modèle simple, à la création d'états d'interface et à l'écart type des fluctuations. Nous avons montré que la prise en compte exclusive de la densité d'états d'interface ne permettait pas d'interpréter les dégradations observées. En effet, seul un modèle dans lequel les états d'interface et les fluctuations de charge sont reliés pour permettre d'interpréter les résultats expérimentaux. Nous pourrons compléter le modèle de dégradation proposé en y incluant la description de la variation du potentiel de bandes plates consécutive à l'injection de charges. Les autres types de modèles proposés dans la littérature reposant sur les 
phénomènes de collision ne permettent pas d'interpréter les fortes dégradations de mobilité observées. Nous avons d'ailleurs négligé l'influence de ces collisions sur la variation de la mobilité $\mu_{0}$.

L'étude expérimentale faite ici montre aussi que les transistors dont le vieillissement a été présenté doivent tous être considérés comme des transistors à canaux très courts auquels sont attachés des phénomènes très particuliers pouvant expliquer que les dégradations les plus importantes apparaissent pour les plus fortes tensions de grille appliquées, ce qui s'oppose aux résultats publiés. Une explication théorique complète de la dégradation est rendue difficile à cause de l'aspect bidimensionnel du transport électrique dans ces TMOS à canaux courts et de l'importance des champs électriques tant latéraux que transversaux. Il faut en effet déterminer et modéliser la fonction de distribution des porteurs chauds dans le TMOS [36].
Quant aux mécanismes de l'injection, leur compréhension a été récemment éclaircie grâce à des travaux sur les capacités MOS [43-45] dans lesquelles la distribution des porteurs est homogène sur toute l'interface $\mathrm{Si} / \mathrm{SiO}_{2}$. Ce problème, déjà complexe, l'est ici encore plus à cause de l'inhomogénéité du champ et celle de la zone dégradée et à cause de la dimension réduite de la surface d'injection.

\section{Remerciements.}

Les auteurs remercient vivement Messieurs S. Cristoloveanu et P. Gentil, ainsi que Messieurs J. J. Niez et G. Reimbold du LETI pour leurs conseils et leurs remarques. Nous tenons à remercier également le Groupement des Circuits intégrés au Silicium (GCIS) pour son soutien financier.

\section{Bibliographie}

[1] Matsumotto, H., Sawada, K., Asai, S., Hirayama, M., Nagasawa, K., IEEE ED 28, 8 (1981) 923.

[2] Hsu, F. C., TAM, S., IEEE EDL 5, 2 (1984) 50.

[3] TAKeda, E., IEE Proc. 131 Part I, 5 (1984) 153.

[4] Chen, K. L., Saller, S. A., Groves, I. A., IEEE ED 32, 2 (1985) 386.

[5] Chenming Hu, Tam, S. C., Hsu, F. C., Ko, P. K., Chan, T. Y., Terrill, K. W., IEEE ED 32, 2 (1985) 375.

[6] TAKeda, E., Shimizu, A., Hagiwara, T., IEEE EDL 4, 9 (1983) 329.

[7] Hofman, K. R., Werner, C., Weber, W., Dorda, G., IEEE ED 32, 3 (1985) 691.

[8] Fischetti, M. V., J. Appl. Phys. 56, 2 (1984) 575.

[9] Tsuchiya, T., Frey, J., IEEE EDL 6, 1 (1985), 8.

[10] Eitan, B., Frohman-BentchKowsky, D., IEEE ED 29, 2 (1982) 254.

[11] Cristoloveanu, S., Cabon-Till, B., Kang, K. N., Gentil, P., Gautier, J., Revue Phys. Appl. 19 (1984) 933.

[12] Sun, S. C., Plummer, J. D., IEEE ED 27, 8 (1980) 1497.

[13] Fang, F. F., Fowler, B., Phys. Rev. 169, 3 (1968) 619.

[14] Chu Hao, Cabon-Till, B., Ghibaudo, G., CristoloVEANU, S., Solid States Electron. 28, 10 (1985) 1025.

[15] Ghibaudo, G., Phys. Status Solidi (1986).

[16] Cabon-Till, B., Ghibaudo, G., Cristoloveanu, S., Electron. Lett. 21, 11 (1985) 457.

[17] TAKEDA, E., SuSUKI, N., IEEE EDL 4, 4 (1983) 111.

[18] RadoJCIC, R., IEEE ED 31, 10 (1984) 1381.

[19] Baglée, D. A., Duvury, C., IEEE EDL 5, 10 (1984) 389.

[20] Overstraeten, R. V., Declerck, G. J., Muls, P. A., IEEE ED 22, 5 (1975) 282.

[21] Muls, P. A., Declerck, G. J., Overstraeten, R. V., Characterisation of the MOSFET operating in weak inversion, Adv. Electron. Electron Phys. 47 (1978) 197.
[22] Brews, J. R., J. Appl. Phys. 46, 5 (1975) 2193.

[23] Arnold, E., Appl. Phys. Lett. 25 (1974) 705.

[24] Ghibaudo, G., Thèse de Docteur ès sciences, INPUSM Grenoble, France (1984).

[25] Yamaguchi, T., Morimoto, S., IEEE ED 30, 6 (1983) 559.

[26] Guebels, P., Van DE Viele, F., Solid State Electrnn. 26, 4 (1983) 267.

[27] Grotjohn, T., Hoefflinger, B., IEEE ED 31, 2 (1984) 234.

[28] Wright, G. T., IEEE ED 32, 7 (1985) 1259.

[29] Pedron, T., Merckel, G., Basset, R., 13th Proc. of MIEL'85, Ljubjana, Yougoslavia (May 1985) 267.

[30] Pedron, T., Denat, G., Merckel, G., Proc. of MIEL'85, Ljubjana, Yougoslavia (May 1985) 245.

[31] SzE, S. M., Physics of semiconductors devices (Wiley Edition, New York) 1982.

[32] Hsu, F. C., Grinolds, H. R., IEEE EDL 5, 3 (1984) 71.

[33] Cabon-Till, B., Ghibaudo, G., Cristoloveanu, S., 15th ESSDERC, Aachen, Germany (Sept. 1985).

[34] Brews, J. R., Physics of the MOS Transistor, Applied Solid States Science, Supplément 2A (Academic Press, New York) 1981.

[35] Katto, H., Muramatsu, S., Solid State Electron. 21 (1978) 459.

[36] KuhNert, R., Werner, C., SChutz, A., IEEE ED 32, 6 (1985) 1057.

[37] Gesch, H., Leburton, J. P., Dorda, G. E., IEEE ED 29, 5 (1982) 913.

[38] Leburton, J. P., Gesch, H., Dorda, G., Solid State Electron. 24, 8 (1981) 763.

[39] Sodini, C. S., Ko, P. K., Moll, J. L., IEEE ED 31, 10 (1984) 1386.

[40] Thurgate, T., Chan, N., IEEE ED 32, 2 (1985) 400.

[41] Eitan, B., Frohman-Bentchkowsky, A., IEEE LD 28, 3 (1981) 328. 
[42] Fang, Z. H., Cristoloveanu, S., Chovet, A., 1/f noise modification by hot electron induced aging in short channel MOSFET's, Proc. Int. Conf. in Noise in Phys. Syst., Roma (Sept. 1985).
[43] Fischetti, M. V., Weinberg, Z. A., Calise, J. A., J. Appl. Phys. 57, 2 (1985) 418.

[44] Fischetti, M. V., Ricco, B., J. Appl. Phys. 57, 8 (1985) 2854.

[45] Fischetti, M. V., J. Appl. Phys. 57, 8 (1985) 2860. 